\title{
Affine Kac-Moody Algebras and Semi-Infinite Flag Manifolds
}

\author{
Boris L. Feigin ${ }^{1}$ and Edward V. Frenkel ${ }^{2, \star}$ \\ 1 117218, Profsoyuznaya, 13/12, apt 91, Moscow, USSR \\ 2 140410, Suvorova, 100, apt 27, Kolomna, Moscow district, USSR \\ Dedicated to Dmitry Borisovich Fuchs on his 50th birthday
}

\begin{abstract}
We study representations of affine Kac-Moody algebras from a geometric point of view. It is shown that Wakimoto modules introduced in [18], which are important in conformal field theory, correspond to certain sheaves on a semi-infinite flag manifold with support on its Schubert cells. This manifold is equipped with a remarkable semi-infinite structure, which is discussed; in particular, the semi-infinite homology of this manifold is computed. The Cousin-Grothendieck resolution of an invertible sheaf on a semi-infinite flag manifold gives a two-sided resolution of an irreducible representation of an affine algebra, consisting of Wakimoto modules. This is just the BRST complex. As a byproduct we compute the homology of an algebra of currents on the real line with values in a nilpotent Lie algebra.
\end{abstract}

\section{Introduction}

In $[18,19]$ we have introduced and studied a new class of representations of affine Kac-Moody algebras, the so-called Wakimoto modules [44]. These representation allow bosonic realization, the Sugawara energy-momentum tensor being quadratic in bosons. This gives a new bosonization rule for the Wess-ZuminoWitten (WZW) models. In [19] we explicitly constructed the intertwining operators between Wakimoto modules and chains (or primary fields) which are submodules of their homomorphisms, using vertex operators. Our results enable us to give an integral representation of the correlation functions in WZW models on the plane in spirit of [14] (it was done soon after [15,27]). In [20] we have proposed the two-sided Bernstein-Gelfand-Gelfand (BGG) resolution, or BRST complex, of an irreducible representation of an affine Kac-Moody algebra, consisting of Wakimoto modules (recall that the usual BGG resolution $[7,26$, $41]$ is one-sided and consists of Verma modules). According to Felder's work [23] (where a similar resolution is constructed over the Virasoro algebra), this

* Address after September 15, 1989: Mathematics Department, Harvard University, Cambridge, MA 02138, USA 
enables us to compute the correlation functions in the WZW models on the torus, "extracting" an irreducible representation from Wakimoto modules with the use of our resolution.

Wakimoto modules also play an important role in the study of the functorial correspondence between affine Kac-Moody algebras and the Virasoro algebra (or $W$-algebras $[20,47])$ and of the highest weight modules with central charge (dual Coxeter number) [19], Appendix B.

Thus we see that Wakimoto modules are very useful both in representation theory and in conformal field theory.

In this work we show that Wakimoto modules naturally appear in the representation theory of affine Kac-Moody algebras: Verma modules, contragradient Verma modules and Wakimoto modules are the particular cases of the general construction. Moreover, modules with similar properties emerge in the case of finite-dimensional simple Lie algebras. Analogous construction seems to exist for arbitrary Kac-Moody algebras.

The crucial point in our investigation is the correspondence between the highest weight representations of the Lie algebra and the sheaves of $\mathscr{D}$-modules (or constructible sheaves) on its flag manifold, lisse with respect to Schubert stratification $[1,2,4,10,34]$.

As well-known, the contragradient Verma module with integral highest weight over a finite-dimensional Lie algebra corresponds to the constant sheaf supported on the big Schubert cell and to the sheaf of local cohomology of the appropriate invertible sheaf on a flag manifold with support on this cell. If we take the local cohomology of the invertible sheaf, with support on another cell, then we obtain the module, which we call the twisted Verma module. Twisted Verma modules with equal highest weights are labelled by elements of the Weyl group (in particular, the unit corresponds to the contragradient Verma module, and the element of maximal length - to the Verma module). Their composition series quotients coincide but the composition series themselves are different.

The Cousin-Grothendieck resolution of a dominant invertible sheaf with respect to Schubert stratification of a flag manifold appears to be the contragradient BGG resolution [35], the Cousin-Grothendieck resolution of a twisted invertible sheaf gives the twisted BGG resolution, which consists of twisted Verma modules. In particular, the element of maximal length of the Weyl group corresponds to the usual BGG resolution.

The infinite-dimensional affine case is more interesting. Here again we have twisted Verma modules, but additional opportunities appear.

The affine Weyl group is infinite and there are no elements of maximal length. But let us consider a "limit element" of "semi-infinite length" of this group. The highest weight module corresponding to is a Wakimoto module. Thus, Wakimoto modules are intermediate between contragradient Verma modules and Verma modules (which correspond to the element of "infinite length").

The sheaves, corresponding to Wakimoto modules, "live" on the limit semiinfinite flag manifold, which is the coset space of the affine Kac-Moody group by an appropriate subgroup. They are lisse with respect to the Schubert stratification. The usual flag manifold of an affine Kac-Moody algebra allows two stratifications: by cells of finite dimension or by cells of finite codimension 
$[40,39]$. The cells of the semi-infinite flag manifold are both of infinite dimension and of infinite codimension.

The semi-infinite flag manifold is endowed with a rather intriguing "semiinfinite structure." For example, its Schubert cells represent the semi-infinite homology classes of this manifold. It is interesting that this homology may also be obtained using Floer's theory [24]. There is a remarkable Morse function of Conley-Zehnder type [11] on this manifold, whose singular points are of semiinfinite indices and give the same semi-infinite homology. We believe that there exists a theory of semi-infinite manifolds, including semi-infinite sheaves and their cohomologies. Our flag manifolds seem to be the first examples of this theory.

A Wakimoto module corresponds to a constant sheaf, supported on a Schubert cell of the semi-infinite flag manifold and to a sheaf of local "semiinfinite cohomology" of the invertible sheaf with support on this cell. CousinGrothendieck resolution is a two-sided BGG resolution (a similar resolution may be obtained in a different way).

The Two-sided BGG resolution gives the semi-infinite analogue of BorelWeil-Bott theorem. As a corollary we obtain an unexpected result about usual homology of the Lie algebras of currents on the real line with values in nilpotent subalgebras of the simple Lie algebra.

The paper is arranged as follows.

In Sect. 2 we treat the finite-dimensional counterpart of our constructions. This section illustrates the main ideas, which we apply later. In Sect. 3 we give the definition of Wakimoto modules over affine Kac-Moody algebras and account for their place in the representation theory of these algebras. Section 4 is devoted to semi-infinite flag manifolds. It clarifies the relations of Wakimoto modules with the geometry of these manifolds. In Sect. 5 we construct Wakimoto modules overcoming certain homological problems. In Sect. 6 we establish and prove twosided BGG resolution and use it for computation of (co)homology. The concluding Sect. 7 is devoted to some examples and applications.

Appendix A contains the definition of semi-infinite (co)homology [16], used in this work. In Appendix B we sketch our results and conjectures [19] about the structure of highest weight modules with central charge - (dual Coxeter number).

\section{The Finite-Dimensional Case}

\subsection{Notations and Preliminaries $[29,5,6]$}

Let $G$ be a complex simple Lie group of rank $n, g$ is its Lie algebra, $n_{-} \oplus h \oplus n_{+}$is the Cartan decomposition of $g, \Delta=\Delta(G)$ is the root system of $G, \Lambda=\Lambda_{G}$ is the root lattice of $G . \Delta=\Delta_{+} \cup \Delta_{-}$, where $\Delta_{+}\left(\Delta_{-}\right)$is the set of positive (negative) roots. Denote by $\alpha_{1}, \ldots, \alpha_{n}$ the set of simple roots, $E_{i}, H_{i}, F_{i}-$ Cartan generators of $g$. Let (,) be the inner product in $h^{*}, S=S(G)$ is the Weyl group of $G, w_{0}$ is its maximal element.

Let $F=G / B$ be flag manifold of $G$, where $B$ is the Borel subgroup of $G$, corresponding to the Lie algebra $b=h \oplus n_{-}$. As is well-known, $F$ decomposes into Schubert cells $F_{s}$, where $s$ runs the Weyl group $S$, which are the orbits of the 
nilpotent group $N_{+}$, corresponding to the Lie algebra $n_{+}$. Namely, $F_{s}=N_{s} \cdot \bar{s}$, where $\bar{s}$ is the image of $s \in G$ under the projection $G \rightarrow F$. The cell $F_{s}$ is isomorphic to $s N_{+} s^{-1} \cap N_{+}, \operatorname{codim} F_{s}=l(s)$ is the length of the element $s$ of the Weyl group, defined as follows: $l(s)=\#\left\{\alpha \in \Delta_{+}: s^{-1} \alpha \in \Delta_{-}\right\}$(here and further \# denotes the number of elements of the set).

The category $\mathcal{O}$ of highest weight representations (in this paper highest weight representation means any representation from the category $\mathcal{O}$ ) of $g$ decomposes into the subcategories $\mathcal{O}_{\theta}$ of representations, where the center of $\mathscr{U}(g)$ acts by the character $\theta$. If $\lambda$ is the dominant integral weight, and $\theta(\lambda)$ is the corresponding central character, then $\mathcal{O}_{\theta(\lambda)}$ consists of the modules with highest weights of the form $s * \lambda=s(\lambda+\varrho)-\varrho, s \in S$, and $\varrho$ is a half of the sum of positive roots of $g$.

\subsection{Twisted Verma Modules}

Let $\mu \in h^{*}$ and $\mathbb{C}_{\mu}$ be one-dimensional representation of $b_{+}=h \oplus n_{+}$, determined as composition $b_{+} \rightarrow h \stackrel{\mu}{\longrightarrow} \mathbb{C}$. Denote by $M_{\mu}$ the Verma module over $g$ with highest weight $\mu: M_{\mu}=\mathscr{U}(g) \underset{\mathscr{U}_{(b+)}}{\otimes} \mathbb{C}_{\mu}$. Verma modules are characterized by the following properties:

a) $M_{\mu}$ lies in the category $\mathcal{O}$,

b) $H_{0}\left(n_{-}, M_{\mu}\right) \simeq \mathbb{C}_{\mu}($ as $h$-module $), H_{i}\left(n_{-}, M_{\mu}\right)=0, i \neq 0$.

Let $M_{\mu}^{*}$ be the contragradient Verma module. It is characterized by the property a) and by the property

b*) $H^{0}\left(n_{+}, M_{\mu}^{*}\right) \simeq \mathbb{C}_{\mu}($ as $h$-module $), H^{i}\left(n_{+}, M_{\mu}^{*}\right)=0, i \neq 0$.

Our aim is to introduce highest weight modules $M_{\mu}^{w}$ over $g$, where $w \in S$ which are intermediate between $M_{\mu}$ and $M_{\mu}^{*}$ in the following sense. Put $n_{+}^{w}=w n_{+} w^{-1}$. $M_{\mu}^{w}$ is characterized by the property a) and by the property

$\left.\mathrm{b}_{\mathrm{w}}\right) H^{l(w)}\left(n_{+}^{w}, M_{\mu}^{w}\right) \simeq \mathbb{C}_{\mu-w(\varrho)+\varrho}($ as $h$-module $)$

$$
H^{i}\left(n_{+}^{w}, M_{\mu}^{w}\right)=0, \quad i \neq l(w) .
$$

In particular, $M_{\mu}^{1} \simeq M_{\mu}^{*}, M_{\mu}^{w_{0}}=M_{\mu}$.

We call $M_{\mu}^{w}$ twisted Verma modules ${ }^{1}$. They are highest weight modules with highest weight $\mu . M_{\mu}^{w}$ is free over $n_{+}^{w} \cap n_{-}$and dual to $M_{\mu}^{w}$ is free over $n_{+}^{w} \cap n_{+}$. Their composition series quotients coincide with those of the Verma module $M_{\mu}$, but they are "glued" in a different way. If $M_{\mu}$ is irreducible then $M_{\mu} \simeq M_{\mu}^{w}$, but if it is not so, then the composition structures of $M_{\mu}^{w_{1}}$ and $M_{\mu}^{w_{2}}$ differ from each other.

We will construct twisted Verma modules.

But first, let us recall the correspondence between the highest weight $g$-modules from $\mathcal{O}_{\theta(\lambda)}(\lambda$ is a dominant integral weight) and certain sheaves on the flag manifold.

Any integral weight $v=w * \lambda$ defines the linear holomorphic complex bundle $\xi_{v}$ on $F$ and invertible sheaf $\tilde{\xi}_{v}$ of its holomorphic sections. Let $\mathscr{D}_{v}$ be the sheaf of differential operators on the sheaf $\xi_{v}$. We denote by $\xi=\widetilde{\xi}_{0}$ the structural sheaf.

${ }^{1}$ Using shifted cohomology $H_{s}^{i}\left(n_{+}^{w}, \cdot\right)$ of $n_{+}^{w}$ with respect to the decomposition $n_{+}^{w}=\left(n_{+}^{w} \cap n_{+}\right) \oplus\left(n_{+}^{w} \cap n_{-}\right)$(see Appendix A) we can rewrite $\left.b_{\mathrm{w}}\right)$ as follows: $H_{s}^{0}\left(n_{+}^{w}, M_{\mu}^{w}\right) \simeq \mathbb{C}_{\mu}$ (as $h$-module), $H_{s}^{i}\left(n_{+}^{w}, M_{\mu}^{w}\right)=0, i \neq 0$ 
The following functor $U_{\lambda}^{w}$ defines the equivalence between the derived category of the highest weight $g$-modules $D \mathcal{O}_{\theta(\lambda)}$ and of the derived category $D_{\lambda}^{w}$ of the $N_{+}$-invariant holonomic $\mathscr{D}_{w * \lambda}$-modules on $F$ [of amplitude $\left.\leqq l(w)\right][1]$

$$
U_{\lambda}^{\omega}: \mathscr{U} \in D_{\lambda}^{\omega} \rightarrow \mathbb{R} \Gamma(F ; \mathscr{U}) \in D \mathcal{O}_{\theta(\lambda)} .
$$

There are also contravariant functors from $D \mathcal{O}_{\theta(\lambda)}$ to the derived category Con of the category Con of constructible sheaves on $F$, lisse with respect to Schubert stratification.

Let $M$ be $g$-module from $\mathcal{O}_{\theta(\lambda)}$. Denote by $\hat{\xi}$ the structural sheaf on the manifold $G / N_{-}$. The sheaf $\hat{\xi} \otimes M$ is equivariant and equipped with the left action of $g$ and the right action of $h$. Consider the complex of sheaves $C_{*}(g, \hat{\xi} \otimes M)$ on $F$, where $C_{*}(g, \hat{\xi} \otimes M)$ denotes the standard homological complex of $g$. This is the complex of sheaves with constructible cohomology, equipped with the action of $h$. So, it decomposes into direct sum:

$$
C_{*}(g, \hat{\xi} \otimes M)=\underset{\mu \in h^{*}}{\oplus} C_{*}^{\mu}(g, \hat{\xi} \otimes M),
$$

where $C_{*}^{\mu}(g, \hat{\xi} \otimes M)$ denotes $\mu$-eigenspace of $h$. The subcomplexes of sheaves $C_{*}^{\mu}(g, \hat{\xi} \otimes M)$ are acyclic if $\mu \neq w * \lambda$. The subcomplex $C_{*}^{w * \lambda}(g, \hat{\xi} \otimes M)$ on $G / N_{-}$ defines the complex of sheaves $\tilde{C}_{*}^{w * \lambda}(M)$ on $F$ and the complex of sheaves with constructible cohomologies $\operatorname{Hom}_{\xi}\left(\widetilde{C}_{*}^{w * \lambda}(M), \tilde{\xi}_{w * \lambda}\right)=\mathscr{V}_{M}$ on $F, \mathscr{V}_{M} \in$ Con. For the point $\kappa \in F$ denote by $b_{\kappa}$ the Lie algebra of the stabilizer of $\kappa$ and by $n_{\kappa}$ its radical. All subalgebras $b_{\kappa}$ are conjugated and we identify $b_{\kappa} / n_{\kappa}=h_{\kappa}$ with $h$. The stalk of $\mathscr{V}_{M}$ at $\kappa$ is isomorphic to $C_{*}^{w * \lambda}\left(n_{\kappa}, M\right)$. This defines the functor $V_{\lambda}^{w}: D \mathcal{O}_{\theta(\lambda)}$ $\rightarrow$ Con.

For a given $\mathscr{V} \in$ DerCon we can construct the $\mathscr{D}_{w * \lambda}$-module using the functor of local cohomology:

$$
Z_{\lambda}^{w}: \mathscr{V} \in \operatorname{Con} \rightarrow \mathbb{R} \operatorname{Hom}_{\xi}\left(\mathscr{V}, \tilde{\xi}_{w * \lambda}\right) \in D_{\lambda}^{w} .
$$

Three functors, defined above, give the following commutative diagram:

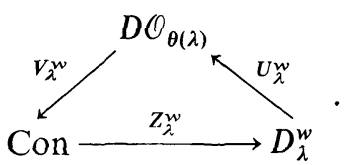

Let $\mathbb{C}_{s}$ be the constant sheaf with support on Schubert cell $F_{s}$. It is well-known that $Z_{\lambda}^{w}\left(\mathbb{C}_{s}\right)=\mathscr{H}_{F_{s}}^{l(s)}\left(F ; \tilde{\xi}_{w * \lambda}\right)[l(s)]$ is the sheaf of local cohomology of $\tilde{\xi}_{w * \lambda}$ with support on $F_{s}$, and so the $g$-module, corresponding to $\mathbb{C}_{s}$ is the space of local cohomology $H_{F_{s}}^{l(s)}\left(F ; \tilde{\xi}_{w * \lambda}\right)$. It follows from the definition, that $H_{F_{s}}^{l(s)}\left(F ; \tilde{\xi}_{w * \lambda}\right)$ is isomorphic to $M_{(s w) * \lambda}^{s}$ (in particular, $H_{F_{1}}^{0}\left(F ; \tilde{\xi}_{w * \lambda}\right) \simeq M_{w * \lambda}^{*}[35], H_{F_{w_{0}}}^{\text {dim } F}\left(F ; \xi_{w * \lambda}\right)$ $\simeq M_{\left(w_{0} w\right) * \lambda}$.

Thus, we see that the twisted Verma module corresponds to the sheaf of local cohomology and to the constant sheaf with support on the Schubert cell. In particular, $V_{\lambda}^{w}\left(M_{(s w) * \lambda}^{s}\right) \simeq \mathbb{C}_{s}$ 

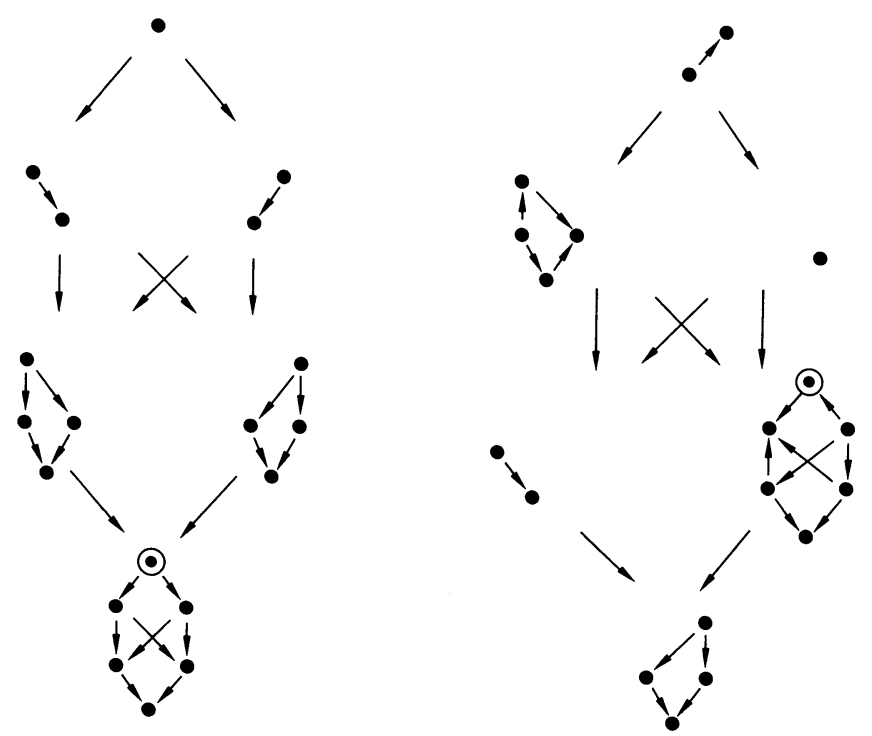

Fig. 1a, b. Generalized BGG resolutions. a Usual BGG resolution. b Twisted BGG resolution

\subsection{Generalized BGG Resolution}

Now we pass to the Cousin-Grothendieck resolution of the invertible sheaf $\xi_{w * \lambda}$. According to the Borel-Weil-Bott theorem [9] $H^{l(w)}\left(F ; \tilde{\xi}_{w * \lambda}\right) \simeq L_{\lambda}$-irreducible representation of $g$ with highest weight $\lambda$ and $H^{i}\left(F ; \tilde{\xi}_{w * \lambda}\right)=0, i \neq l(w)$.

The spectral sequence for the computation of $H^{i}\left(F ; \xi_{w * \lambda}\right)$ associated with the filtration of $F$ by Schubert varieties degenerates in the first term and we obtain the Cousin-Grothendieck resolution $C_{w * \lambda}^{*}$, whose terms are isomorphic to

$$
C_{w * \lambda}^{j}=\underset{\substack{s \in S \\ l(s)=j}}{\oplus} H_{F_{s}}^{l(s)}\left(\tilde{\xi}_{w * \lambda}\right)=\underset{\substack{s \in S \\ l(s)=j}}{\oplus} M_{(s w) * \lambda}^{s} .
$$

The cohomologies of this resolution are trivial in all dimensions except the $l(w)^{\text {th }}$ and the $l(w)^{\text {th }}$ cohomology is isomorphic to $L_{\lambda}$.

The complex $C_{\lambda}^{*}$ (investigated in [35]) is contragradient to the BGG resolution [7] and consists of contragradient Verma modules. The complex $C_{w_{0} * \lambda}^{*}$ coincides with BGG resolution and consists of Verma modules.

We call $C_{w * \lambda}^{*}$ the generalized strong $B G G$ resolution. It consists of twisted Verma modules, and its $l(w)^{\text {th }}$ cohomology group is isomorphic to irreducible representation $L_{\lambda}$ of $g$. The structures of the usual and one of the twisted BGG resolutions over $s l_{3}$ are shown in Fig. 1.

\subsection{Algebraic Constructions}

Now we will give algebraic constructions of twisted Verma modules and generalized BGG resolutions. They will be adapted in Sect. 5 for construction of Wakimoto modules. 
Let $\Gamma_{w}$ be the Heisenberg algebra with basis $a_{\alpha}, a_{\alpha}^{*}, \alpha \in w\left(\Delta_{+}\right)=\Delta_{+}^{w}$ and commutation relations $\left[a_{\alpha}, a_{\beta}\right]=\left[a_{\alpha}^{*}, a_{\beta}^{*}\right]=0,\left[a_{\alpha}, a_{\beta}^{*}\right]=\delta_{\alpha, \beta}$. Let $\pi_{w}$ be an irreducible representation of $\Gamma_{w}$ with vacuum vector, annihilated by $a_{\alpha}, \alpha \in \Delta_{+}^{w} \cap \Delta_{+}$and by $a_{\alpha}^{*}, \alpha \in \Delta_{+}^{w} \cap \Delta_{-}$. Denote by $A_{0}$ the algebra of operators on $\pi_{w}$, commuting with $a_{\alpha}^{*}, \alpha \in \Delta_{+}^{w}$ (it consists of all polynomials on $a_{\alpha}^{*}, \alpha \in \Delta_{+}^{w}$ ) and by $A_{1}$-its normalizer. Evidently, $A_{1} / A_{0}$ is identified with Lie algebra Vect ${ }_{w}$ of vector fields on the formal neighbourhood $\mathscr{U}_{w}$ of the cell $F_{w} \subset F$. Lie algebra $g$ acts on $\mathscr{U}_{w}$ and hence imbeds into Vect ${ }_{w}$, the space of embeddings being parametrized by $H^{1}\left(g, A_{0}\right) \simeq h^{*}$. Fix $\mu \in h^{*}$ and the corresponding embedding. Then $\pi_{w}$ is $g$-module with highest weight $\mu$, which is isomorphic to $M_{\mu}^{w}$. In particular, if $w=1$, then $M_{\mu}^{w}$ is the module of the functions on the big cell of $F$, which is isomorphic to the contragradient Verma module $M_{\mu}^{*}$, if $w=w_{0}$, then $M_{\mu}^{w}$ is the module of $\delta$-functions on $F$, isomorphic to Verma module $M_{\mu}$.

Now we will give a description of the generalized weak $B G G$ resolutions.

Let $\Omega_{w}^{*}$ be the de Rham complex on the formal neighbourhood $\mathscr{U}_{w}$ of the cell $F_{w}$. Consider the corresponding complex $\widetilde{\Omega}_{w}^{*}$ of local cohomologies:

$$
H_{F_{w}}^{l(w)}\left(\mathscr{U}_{w}, \Omega_{w}^{0}\right) \rightarrow H_{F_{w}}^{l(w)}\left(\mathscr{U}_{w}, \Omega^{1}\right) \rightarrow H_{F_{w}}^{l(w)}\left(\mathscr{U}_{w}, \Omega^{2}\right) \rightarrow \ldots
$$

Evidently, cohomologies of this complex are non-trivial only in dimension $l(w)$ and equal to $\mathbb{C}$. Consider the tensor product complex $L_{\lambda} \otimes \widetilde{\Omega}_{w}^{*}=\Omega_{w}^{*}(\lambda)$. The center of $\mathscr{U}(g)$ acts on $\widetilde{\Omega}_{w}^{*}(\lambda)$ and $\Omega_{w}^{*}(\lambda)$ decomposes into a direct sum of subcomplexes, corresponding to eigenvalues $\theta$ of the center: $\Omega_{w}^{*}(\lambda)=\oplus \Omega_{w}^{*}(\lambda)_{\theta}$. The subcomplexes $\Omega_{w}^{*}(\lambda)_{\theta}$ are acyclic if $\theta \neq \theta(\lambda)$. The subcomplex $\Omega_{w}^{*}(\lambda)_{\theta(\lambda)}$ is the generalized weak BGG resolution $\bar{C}_{w * \lambda}^{*}$. For $w=1$ this fact was proved in [7]. In other cases the proof is analogous. $\bar{C}_{w * \lambda}^{*}$ is isomorphic to $C_{w * \lambda}^{*}$ only if $w=1$ or $w=w_{0}$.

We give algebraic construction of this complex.

Let $T^{+}\left(\mathscr{U}_{w}\right)$ be the tangent bundle over $\mathscr{U}_{w}$ with changed parity of fibers. The complex $\Omega_{w}$ is the restriction to $g$ of the graded module over the Lie superalgebra of vector fields on $T^{+}\left(\mathscr{U}_{w}\right)$, which contains the canonical element-differential, commuting with $g$.

Let $\Gamma_{w}^{+}$be the extension of $\Gamma_{w}$ by odd generators $\varphi_{\alpha}, \varphi_{\alpha}^{*}, \alpha \in \Delta_{+}^{w}$ commuting with $\Gamma_{w}$ and with the following anti-commutation relations:

$$
\left[\varphi_{\alpha}, \varphi_{\beta}\right]_{+}=\left[\varphi_{\alpha}^{*}, \varphi_{\beta}^{*}\right]_{+}=0, \quad\left[\varphi_{\alpha}, \varphi_{\beta}^{*}\right]_{+}=\delta_{\alpha, \beta} .
$$

Let $\pi_{w}^{+}$be the irreducible representation of $\Gamma_{w}^{+}$with vacuum vector, annihilated by $a_{\alpha}, \varphi_{\alpha}, \alpha \in \Delta_{+}^{w} \cap \Delta_{+}$and by $a_{\alpha}^{*}, \varphi_{\alpha}^{*}, \alpha \in \Delta_{+}^{w} \cap \Delta_{-}$. Introduce grading on $\Gamma_{w}^{+}$and $\pi_{w}^{+}$, putting $\operatorname{deg} a_{\alpha}=\operatorname{deg} a_{\alpha}^{*}=0, \operatorname{deg} \varphi_{\alpha}=-1, \operatorname{deg} \varphi_{\alpha}^{*}=1$.

Denote by $A_{0}^{+}$the algebra of operators on $\pi_{w}^{+}$commuting with $a_{\alpha}, \varphi_{\alpha}, \alpha \in \Delta_{+}^{w}$, and let $A_{1}^{+}$be its normalizer in End $\pi_{w}^{+} . A_{1}^{+} / A_{0}^{+}$is identified with Lie superalgebra of vector fields on $T^{+}\left(\mathscr{U}_{w}\right)$, and since $g$ acts on $T^{+}\left(\mathscr{U}_{w}\right)$, there is the embedding of $g$ into this superalgebra.

There is a canonical element $d \in \Gamma_{w}^{+}$of degree 1 such that $[d, g]=0,[d, d]_{+}=0$. This equips $\pi_{w}^{+}$with the structure of the complex of $g$-modules, which is isomorphic to $\widetilde{\Omega}_{w}^{*}$. Note that if $w=1$, then $\pi_{w}^{+}$and $\widetilde{\Omega}_{w}^{*}$ is nothing but the de Rham complex on the big cell of $F$. 
Using this algebraic construction we can describe the generalized BGG resolution. Introduce modified length on Weyl group. Let

$$
\varphi_{s}=\left\{\alpha \in \Delta_{+}: s^{-1} \alpha \in \Delta_{-}\right\}
$$

and let $\Delta_{( \pm)}^{w}=w\left(\Delta_{ \pm}\right) \cap \Delta_{+}$. Put

$$
l_{w}(s)=\#\left(\varphi_{s} \cap \Delta_{(+)}^{w}\right)-\#\left(\varphi_{s} \cap \Delta_{(-)}^{w}\right) .
$$

In particular, $l_{1}(s)=l(s), l_{w_{0}}(s)=-l(s)$.

We have $\bar{C}_{w * \lambda}^{i+l(w)}=\underset{\substack{s \in S \\ l_{w}(s)=i}}{\bigoplus} M_{s * \lambda}^{w}$. Using this result we can compute the cohomology $H^{*}\left(n_{+}^{w}, L_{\lambda}\right)$. Namely,

$$
H^{i+l(w)}\left(n_{+}^{w}, L_{\lambda}\right)=\underset{\substack{s \in S \\ l_{w}(s)=i}}{\bigoplus} \mathbb{C}_{s * \lambda-w(\varrho)+\varrho}
$$

as the $h$-module, or equally, $H_{s}^{i}\left(n_{+}^{w}, L_{\lambda}\right)=\underset{\substack{s \in S \\ l_{w}(s)=i}}{\oplus} \mathbb{C}_{s * \lambda}$. This is the Borel-Weil-Bott [9] theorem.

In particular, $\operatorname{dim} H^{i}\left(n_{+}^{w}, L_{\lambda}\right)=\#\{s: l(s)=i\}$ for any $w$. We can compare it with the following result:

$$
\operatorname{dim} H^{2 i}(F ; \mathbb{C})=\#\{s: l(s)=i\}, \quad H^{2 i+1}(F ; \mathbb{C})=0 .
$$

We will generalize this result to semi-infinite flag manifolds in Sect. 4.

In conclusion we define certain functors in the category $\operatorname{Der} \mathcal{O}_{\theta(\lambda)}$ connected with the Weyl group. Let $T_{w}^{s}=U_{\lambda}^{s} \circ Z_{\lambda}^{s} \circ V_{\lambda}^{w}$. We have $T_{w}^{s} \circ T_{s^{\prime}}^{w}=T_{s^{\prime}}^{s}$. The functor $T_{w}^{s}$ transforms the twisted Verma module $M_{\left(s^{\prime} w\right) * \lambda}^{w}$ to $M_{\left(s^{\prime} s\right) * \lambda}^{s}$. So $T_{w}^{s}$ transforms the generalized BGG resolution $C_{w * \lambda}^{*}$ to $C_{s * \lambda}^{*}$, and hence $L_{\lambda}$ to $L_{\lambda}[l(s)-l(w)]$. Note, that Bruhat-Hecke correspondence $N_{w}$ on

$$
F \times F: N_{w}=\left\{\left(\kappa, \kappa^{\prime}\right) \in F \times F:\left(b_{\kappa}, b_{\kappa^{\prime}}\right) \text { are in relative position } w\right\}
$$

gives the functor $T_{1}^{w}=U_{\lambda}^{1} \circ N_{w^{*}} \circ Z_{\lambda}^{1} \circ V_{\lambda}^{1}$. Functors $T_{w}^{\mathrm{s}}$ seem to be closely related to Kazhdan-Lusztig theory [33]. It would be interesting to give an algebraic construction for them.

\section{Wakimoto Modules: Definition}

Now we pass to representations of affine Kac-Moody algebras. The affine KacMoody algebra $\mathrm{Lg}^{\Lambda}$ is the unique central extension of the algebra of currents $L g=g \otimes \mathbb{C}((t))$. The commutation relations in $L g^{\Lambda}$ read

$$
[A(m), B(l)]=[A, B](m+l)+m \cdot \delta_{m,-l}\langle A, B\rangle \cdot K,
$$

where $A(m)$ denotes $A \otimes t^{m} \in L g, A \in g, K$ is the central element, $\langle$,$\rangle is Killing form,$ normalized so that $\left\langle H_{i}, H_{j}\right\rangle=\left(\bar{\alpha}_{i}, \bar{\alpha}_{j}\right)$, where $\bar{\alpha}_{i}=2 \alpha_{i} /\left(\alpha_{i}, \alpha_{j}\right), i=1, \ldots, n$.

Let $\hat{\Delta}$ be the root system of $L g^{\Lambda}, \alpha_{0}, \alpha_{1}, \ldots, \alpha_{n}$ be the set of simple roots, $\hat{\Delta}_{+}\left(\hat{\Delta}_{-}\right)$ denotes the set of positive (negative) roots. The roots of $\mathrm{Lg}^{\Lambda}$ are divided into imaginary and real roots. Imaginary roots are of the form $l \delta$, where $l \in \mathbb{Z}$ and $\delta=\alpha_{0}$ 
$+\alpha_{\max }, \alpha_{\max }$ being maximal root of $g$. Real roots are of the form $l \delta+\alpha$, where $l \in \mathbb{Z}$, $\alpha \in \Delta$.

Cartan decomposition of $L g^{A}$ is given by $L g^{A}=\hat{n}_{-} \oplus \hat{h} \oplus \hat{n}_{+}$, where

$$
\begin{gathered}
\hat{n}_{-}=n_{-} \otimes 1 \oplus g \otimes t^{-1} \mathbb{C}\left[t^{-1}\right], \quad \hat{h}=h \otimes 1 \oplus \mathbb{C} K, \\
\hat{n}_{+}=n_{+} \otimes 1 \oplus g \otimes t \mathbb{C}[[t]] .
\end{gathered}
$$

Any character $\chi$ of $\hat{h}$ determines the character of the algebra $\hat{b}_{+}=\widehat{h} \oplus \hat{n}_{+}$, $\hat{b}_{+} \rightarrow \widehat{h}_{+} \stackrel{\chi}{\longrightarrow} \mathbb{C}\left[\right.$ note, that $\chi=(\bar{\chi}, k), \bar{\chi} \in h^{*}, k \in(\mathbb{C} K)^{*} \simeq \mathbb{C}, k$ is called the central charge]. Let $M_{\chi}$ be Verma module $\mathscr{U}\left(L g^{\Lambda}\right) \otimes \mathbb{C}_{\chi}$, where $\mathbb{C}_{\chi}$ is one-dimensional representation of $\hat{b}_{+}$, determined by $\chi$.

The category $\hat{\mathcal{O}}$ of highest weight $L g^{A}$-modules $[13,41]$ decomposes into the direct sum of subcategories $\hat{\mathcal{O}}_{\theta}$, where $\theta$ is the eigenvalue of the Casimir element of $L g^{\Lambda}[29,30]$. Denote by $\hat{\mathcal{O}}_{\theta(\chi)}$ the subcategory containing $M_{\chi}[\theta(\chi)=(\chi+2 \varrho, \chi)$, where $\left.\varrho \in h^{*}, \varrho\left(\alpha_{i}\right)=1, i=0, \ldots, n\right]$. In particular, if $\chi$ is the dominant integral, then $\hat{\mathcal{O}}_{\theta(x)}$ consists of modules with highest weights of the form $s * \chi=s(\chi+\varrho)-\varrho$, where $s$ is an element of affine Weyl group $S_{\text {aff }}=S_{\text {aff }}(G)$.

The Verma module $M_{\chi}$ is characterized by the following properties (cf. Sect. 2):

a) $M_{\chi}$ belongs to the category $\hat{\mathcal{O}}$,

b) $H_{0}\left(\hat{n}_{-}, M_{\chi}\right) \cong \mathbb{C}_{\chi}($ as $\hat{h}$-module $), H_{i}\left(\hat{n}_{-}, M_{\chi}\right)=0, i \neq 0$.

Now let $M_{\chi}^{*}$ be a module, contragradient to $M_{\chi}$. $M_{\chi}^{*}$ is characterized by the property a) and by the property

$\left.\mathrm{b}^{*}\right) H^{0}\left(\hat{n}_{+}, M_{\chi}^{*}\right) \simeq \mathbb{C}_{\chi}($ as $\hat{h}$-module $), H^{i}\left(n_{+}, M_{\chi}^{*}\right)=0, i \neq 0$.

Following Sect. 2 we should consider the flag manifold $X=X(G)=L G^{\Lambda} / B^{\Lambda}$ $[39,40]$. Here $L G^{\Lambda}$ denotes the unique central extension of the group of all smooth maps $S^{1} \rightarrow G$ and $B^{A}$ denotes Lie subgroup of $L G^{A}$ corresponding to Lie algebra $\hat{b}=\hat{n}_{-} \oplus \hat{h}$. We also need the dense submanifold $X_{\mathrm{an}}=X_{\mathrm{an}}(G)$ of $X, X_{\text {an }}$ $=L_{\mathrm{an}} G^{\Lambda} / B_{\mathrm{an}}^{\Lambda}$, where $L_{\mathrm{an}} G^{\Lambda}$ denotes the group of analytic maps $S^{1} \rightarrow G$, and $B_{\mathrm{an}}^{\Lambda}$ denotes the corresponding subgroup. Flag manifold $X$ decomposes into disjoint union of Schubert cells $X_{s}$, where $s$ runs the affine Weyl group $S_{\text {aff }}$ of $G$, which are the orbits of $\hat{N}_{+}$(Lie group of $\hat{n}_{+}$). $X_{s}=\hat{N}_{+} \cdot \bar{s}$, where $\bar{s}$ is the image of $s \in L G^{\Lambda}$ in $X$. The Schubert cell $X_{s}$ is isomorphic to $s \hat{N}_{+} s^{-1} \cap \hat{N}_{+}$, and is of finite codimension.

Let $\chi$ be integral dominant weight. Then we can introduce the functors $\hat{U}_{\lambda}^{w}, \hat{V}_{\lambda}^{w}, \hat{Z}_{\lambda}^{w}$, acting between the appropriate derived categories. It is possible to construct twisted Verma modules $M_{(s w) * \chi}^{s}$ as local cohomology $H_{X_{s}}^{l(s)}\left(X, \xi_{w * \chi}\right)$, where $\tilde{\xi}_{w * \chi}$ denotes the suitable invertible sheaf on $X$. Twisted Verma modules $M_{\mu}^{w}$ are generally characterized by the property

$$
\begin{aligned}
&\left.\mathrm{b}_{\mathrm{w}}\right) \quad H^{l(w)}\left(w \hat{n}_{+} w^{-1}, M_{\mu}^{w}\right) \simeq \mathbb{C}_{\mu-w(\varrho)+\varrho}, \\
& H^{i}\left(w \hat{n}_{+} w^{-1}, M_{\mu}^{w}\right)=0, \quad i \neq l(w) .
\end{aligned}
$$

Here $l(w)=\#\left\{\alpha \in \hat{\Delta}_{+}: w^{-1} \alpha \in \hat{\Delta}_{-}\right\}$is the length of $w \in S_{\text {aff. }}$. In particular, $M_{\mu}^{1}$ is the contragradient Verma module $M_{\mu}^{*}$.

Twisted Verma modules correspond to constant sheaves, supported on the Schubert cell. It is possible to construct them algebraically and also to define affine Weyl twist functors $T_{w}^{s}$ as in 2.4. Twisted Verma modules compose twisted BGG resolutions of irreducible representation as in 2.3. 
So our program may be generalized in the affine case as well. But in contrast to the finite-dimensional case, the affine Weyl group is infinite and there are no maximal elements. That is why, starting with the contragradient Verma module $M_{\mu}^{*}$ we cannot pass to the Verma module $M_{\mu}$ "moving" on the Schubert cell, as in the finite-dimensional case (where $M_{\mu}^{*}$ corresponds to the cell of maximal dimension and $M_{\mu}$ to the cell of maximal codimension - to the point), in the affine case there are no Schubert cells of maximal codimension.

But we can try to take into consideration twisted Verma modules, which correspond to certain "limit elements" of the affine Weyl group (and also "limit" cells of flag manifold).

Recall that $S_{\text {aff }} \simeq S \propto \Lambda$, where $S$ is the finite Weyl group and $\Lambda$ is the root lattice.

The group $S_{\text {aff }}$ acts on $L g^{\Lambda}$ as follows:

$$
s \cdot t_{a}\left(X_{\alpha} \otimes t^{l}\right)=X_{s(\alpha)} \otimes t^{l+(a, \alpha)}, \quad \alpha \in \Delta, s \in S, a \in \Lambda .
$$

Choose an element $\gamma \in \Lambda$ and transform the subalgebra $\hat{n}_{+}$by its powers:

$$
t_{m \gamma}\left(X_{\alpha} \otimes t^{l}\right)=X_{\alpha} \otimes t^{l+m(\alpha, \gamma)},
$$

and so when $m \rightarrow \infty$ we obtain the limit subalgebra $\hat{n}_{+}(\gamma)=\lim t_{m \gamma}\left(\hat{n}_{+}\right)$:

$$
\hat{n}_{+}(\gamma)=\underset{\substack{\alpha \in \Delta \\(\alpha, \gamma)<0}}{\oplus} \mathbb{C} X_{\alpha} \otimes \mathbb{C}((t)) \oplus\left(h \oplus \underset{\substack{\alpha \in \Delta \\(\alpha, \gamma)=0}}{\oplus} \mathbb{C} X_{\alpha}\right) \otimes t \mathbb{C}[[t]] \oplus \underset{\substack{\alpha \in \Delta_{ \pm} \\(\alpha, \gamma)=0}}{\oplus} \mathbb{C} X_{\alpha} \otimes 1 .
$$

Note that $p_{\gamma}=h \oplus \underset{\substack{\alpha \in A \\(\alpha, \gamma) \leq 0}}{\oplus} \mathbb{C} X_{\alpha}$ is the parabolic subalgebra of $g$.

The algebra $\hat{n}_{+}(\gamma)$ depends only on $p_{\gamma}$, so we denote $a_{p}=\hat{n}_{+}(\gamma)$, where $p=p_{\gamma}$. Let $p=r_{p} \oplus v_{p}$, where $v_{p}$ is the reductive subgroup of $p$ and $r_{p}$ is the nilpotent radical of $p$. Then

$$
a_{p}=r_{p} \otimes \mathbb{C}((t)) \oplus v_{p} \otimes t \mathbb{C}[[t]] \oplus\left(v_{p} \cap n_{+}\right) \otimes 1 .
$$

We have the twisted Cartan decomposition of $L g^{\Lambda}: L g^{\Lambda}=a_{p} \oplus \widehat{h} \oplus a_{p}^{*}$, where * denotes Cartan involution [29].

It is natural to consider the infinitely twisted Verma modules $W_{\chi, p}$, corresponding to $a_{p}$ (and to $\lim t_{m \gamma}$ ) which are characterized by the property a) and by the property

$$
\begin{array}{lll}
\left.\mathrm{b}_{\mathrm{p}}\right) & H^{\infty / 2+i}\left(a_{p}, W_{\chi, p}\right)=0, & i \neq 0, \\
& H^{\infty / 2}\left(a_{p}, W_{\chi, p}\right) \simeq \mathbb{C}_{\chi} & (\text { as } \hat{h} \text {-module })
\end{array}
$$

where $H^{\infty / 2+i}\left(a_{p}, \cdot\right)$ denotes semi-infinite cohomology of $a_{p}$ with respect to decomposition $a_{p}=a_{p}^{+} \oplus a_{p}^{-}, a_{p}^{ \pm}=a_{p} \cap \hat{n}_{ \pm}$(see Appendix A).

We call $W_{x, p}$ Wakimoto modules (note, that in [18-20] we considered only $W_{\chi, b}$. Composition series quotients of $W_{\chi, p}$ and $M_{\chi}$ coincide, and if $M_{\chi}$ is irreducible, then $M_{\chi}$ is isomorphic to $W_{\chi, p}$. $W_{\chi, p}$ is free over $a_{p}^{-}$and dual to $W_{\chi, p}$ is free over $a_{p}^{+}$.

One can define $W_{\chi, p}$ as the limit of $M_{\chi}^{t_{m y}}$ in the sense of Jantzen filtration [30]. Let $J^{i} W_{\chi, p}$ and $J^{i} M_{\chi}^{t_{m y}}$ be the $i^{\text {th }}$ terms of Jantzen filtration of $W_{\chi, p}$ and $M_{\chi}^{t_{m y}}$. Then for any $j$ there is $m_{0}$ such that $J^{i} W_{\chi, p} \simeq J^{i} M_{\chi}^{t_{m y}}$ for $i=1, \ldots, j, m>m_{0}$. 
According to our geometric approach, we should consider the semi-infinite flag manifold $X_{p}=L G^{\Lambda} / B_{p}^{\Lambda}=L G / B_{p}$, where $B_{p}^{\Lambda}$ is the twisted Borel subgroup corresponding to Lie algebra $\hat{b}_{p}=a_{p}^{*} \oplus \hat{h}$ (twisted Borel subalgebra) and its dense submanifold $X_{p}^{\text {an }}=L_{\mathrm{an}} G^{\Lambda} / B_{p}^{\Lambda_{\mathrm{an}}}=L_{\mathrm{an}} G / B_{p}^{\mathrm{an}}$.

In the next section we will study the geometrical and topological properties of $X_{p}, X_{p}^{\text {an }}$. In particular, we will show that $X_{p}^{\text {an }}$ decomposes into Schubert cells of semi-infinite dimensions (that is both of infinite dimension and of infinite codimension), which are labelled by the affine Weyl group. These cells are related to Wakimoto modules in the same way as cells on $X$ are related to contragradient Verma modules (or twisted Verma modules): the constant sheaf on the cell corresponds to Wakimoto module, and the Wakimoto module is isomorphic to the local "semi-infinite" cohomology of the invertible sheaf on $X_{p}$. CousinGrothendieck resolution of the invertible sheaf (with respect to Schubert stratification) gives two-sided BGG resolution of the irreducible representation, whose terms are Wakimoto modules. Explicit algebraic constructions of Wakimoto modules and of two-sided BGG resolutions (in spirit of 2.4) are given in Sects. 5 and 6.

Recall that we have defined Wakimoto modules starting with the contragradient Verma module $M_{\mu}^{*}$ and taking the limit element on the affine Weyl group. It is also possible to start with Verma module $M_{\mu}$. In this way we obtain co-twisted Verma modules, characterized by the property:

$$
\begin{aligned}
\left.b^{w}\right) & H_{l(w)}\left(w \hat{n}_{-} w^{-1},{ }^{w} M_{\mu}\right) \simeq \mathbb{C}_{\mu+\varrho-w(\varrho)} \quad(\text { as } h \text {-module }), \\
& H_{i}\left(w \hat{n}_{-} w^{-1},{ }^{w} M_{\mu}\right)=0, \quad i \neq l(w) .
\end{aligned}
$$

Evidently, ${ }^{w} M_{\mu} \cong M_{\mu}^{w *}$.

These modules correspond to the sheaves on the "turned" flag manifold $X^{+}=L G^{A} / B_{+}^{A}$, where $B_{+}^{A}$ is the Lie group corresponding to $\hat{b}_{+}$(or on its dense submanifold $X_{\mathrm{an}}^{+}=L_{\mathrm{an}} G / B_{+}^{\Lambda_{\mathrm{an}}}$ ). These sheaves are supported on Schubert cells $X_{s}^{+}$ of $X^{+}: X_{s}^{+}=\hat{N}_{+} \cdot \bar{s}$. The cell $X_{s}^{+}$is isomorphic to $s \hat{N}_{-} s^{-1} \cap \hat{N}_{+}$and is of finite dimension.

Taking the limit, as above, we obtain contragradient Wakimoto modules $W_{\chi, p}^{*}$, corresponding to limit elements of $S_{\text {aff }}$ (and to limit cells of $X^{+}$). They are characterized by the property

$$
\begin{aligned}
\left.\mathrm{b}^{\mathrm{p}}\right) & H_{\infty / 2+i}\left(a_{p}^{*}, W_{\chi, p}^{*}\right)=0, \quad i \neq 0, \\
& H_{\infty / 2}\left(a_{p}^{*}, W_{\chi, p}^{*}\right) \simeq \mathbb{C}_{\chi} \quad(\text { as } h \text {-module }),
\end{aligned}
$$

where $H_{\infty / 2+i}\left(a_{p}^{*}, \cdot\right)$ denotes semi-infinite homology of $a_{p}^{*}$ with respect to the decomposition: $a_{p}^{*}=a_{p}^{*+} \oplus a_{p}^{*-}, a_{p}^{* \pm}=a_{p}^{*} \cap \hat{n}_{ \pm}$.

The corresponding flag manifold is $X_{p}^{+}=L G^{\Lambda} / B_{p}^{\Lambda+}$, where $B_{p}^{\Lambda_{+}}$is the Lie group corresponding to the Lie algebra $\hat{b}_{p}^{+}=a_{p} \oplus \hat{h}$.

We see that the way starting with $M_{\mu}$ is dual to the way starting with $M_{\mu}^{*}$. Possibly, there are other ways.

Wakimoto modules are intermediate between $M_{\mu}$ and $M_{\mu}^{*}$. The cells of $X_{p}, X_{p}^{+}$ may be considered as infinitely far cells of the usual flag manifolds $X$ and $X^{+}$.

So we may consider manifolds $X, X_{p}, X_{p}^{+}, X^{+}$as the pieces of the unified "flag manifold" of affine Kac-Moody algebra $\mathrm{Lg}^{\Lambda}$, whose geometry is in one-to-one correspondence with representation theory of $L g^{\Lambda}$. 


\section{Semi-Infinite Flag Manifolds}

In this section we will study semi-infinite flag manifolds $X_{p}$ and their submanifolds.

\subsection{The Usual Cohomology of $X_{p}$}

The group $B_{p}^{A}$ is homotopically equivalent to the group $\hat{H}$ (Lie group of $\hat{h}$ ) for any $p$. Hence $L G^{\Lambda} / B_{p}^{\Lambda}$ is homotopically equivalent to $L G^{\Lambda} / \hat{H} \simeq L G / H$ and to $\varphi=\widetilde{\mathscr{U}} / \widetilde{T}$, where $\tilde{\mathscr{U}}$ is a compact form of $L G$ and $\widetilde{T}$ is its maximal torus, acting on $\widetilde{\mathscr{U}}$ as constant maps. So we obtain the following.

Proposition 1. For any $p$ the semi-infinite flag manifold $X_{p}$ is homotopically equivalent to the manifold $X=L G^{\Lambda} / \hat{B}$. In particular, the cohomology rings of $X_{p}$ and $X$ coincide, and

$$
\operatorname{dim} H^{2 i}\left(X_{p}, \mathbb{Z}\right)=\#\left\{s \in S_{\text {aff }}: l(s)=i\right\}, \quad H^{2 i+1}\left(X_{p}, \mathbb{Z}\right)=0 .
$$

Note, that $H^{*}(X, \mathbb{Z})$ was computed on $[36,31, \ldots]$.

\subsection{Loop Spaces and Semi-Infinite Structure}

Let $R_{p}$ be the nilpotent radical of $P$ (Lie group of $p$ ) and $N_{p}=P / R_{p}$ the reductive subgroup of $P . V_{p}$ is the product of the semi-simple Lie group $G_{p}$ and abelian group $H_{p}$.

Consider the space $L(P)$ of $C^{\infty}$-maps $S^{1} \rightarrow F_{p}=G / P$. Note that $\pi_{1}(L(P)) \simeq H_{2}\left(F_{p}, \mathbb{Z}\right)$ is isomorphic to the group of characters $P \rightarrow \mathbb{C}^{*}$ (or the quotient of lattices $\Lambda_{G} / \Lambda_{G_{p}}$. Denote by $\widetilde{L(P)}$ the universal covering space of $L(P)$. This space is isomorphic to the space of $C^{\infty}$-maps $D^{2} \rightarrow F_{p}$, where $D^{2}$ is a closed disk, up to the following equivalence: two maps $\gamma_{1}: D^{2} \rightarrow F_{p}$ and $\gamma_{2}: D^{2} \rightarrow F_{p}$ are identified, if they coincide on the border of $D^{2}$ and are homotopically equivalent in the class of such maps.

The natural map $X_{p} \rightarrow L(P)$ is a fibering, the fiber being the usual (not semiinfinite) flag manifold $Y_{p}$ of the group $L V_{p}^{\Lambda} . Y_{p}$ is the product of $X\left(G_{p}\right)$ and $X\left(H_{p}\right)$, where $X\left(H_{p}\right)$ - "flag manifold" of $L H_{p}^{\Lambda}$ - is isomorphic to the product of the group of characters of $P$ [or to $\left.H_{2}\left(F_{p}, \mathbb{Z}\right)\right]$ and the vector space $h_{p} \otimes t \mathbb{C}[t]\left(h_{p}\right.$ is the Lie algebra of $\left.H_{p}\right)$. Thus, $X_{p}$ is homotopically equivalent to the bundle over $\overparen{L(P)}$ with fiber $X\left(G_{p}\right)$. Denote it by $\bar{X}_{p}$.

Note that $\widetilde{L(P)} \simeq L G / L P_{0}$, where $L P_{0}$ denotes the connected component of the unit of the group $L P$. We put $\widetilde{L_{a n}}(P)=L_{\mathrm{an}} G / L_{\mathrm{an}} P_{0}$.

The manifolds $L(P)$ and $L_{\mathrm{an}}(P)$ are endowed with remarkable semi-infinite structure. At this moment we cannot give a strict definition of such objects as semiinfinite manifolds, sheaves on them and semi-infinite cohomology of sheaves. But we are convinced that the suitable theory does exist and that manifolds $\widetilde{L(P)}, \widetilde{L_{a n}}(P)$ are the first examples of this theory.

As an illustration we will compute the semi-infinite homology of these manifolds. We will propose two ways for computations.

The first one is an application of Floer's theory [24] which is the semi-infinite analogue of the usual Morse theory [8]. In contrast to the usual theory in Floer's theory the indices of the singular points of the Morse function are infinite, but the difference of indices of two points is finite, so we can define the relative index 
putting the index of a certain marked point to be equal to 0 . Singular points compose Morse complex graded by the relative index with a differential defined as usual and semi-infinite homologies are the homologies of this complex. In our case there is a remarkable Morse function of Conley-Zehnder type [11], the complex being non-trivial only in "even" dimensions (as in finite-dimensional case [8]), and so singular points represent homology classes of $\overline{L(P)}$.

The second is the decomposition of $\widetilde{L_{\text {an }}}(P)$ into Schubert cells of semi-infinite dimensions. It means that cells are both of infinite dimension and of infinite codimension, but they are commensurable, so that we can define the relative dimension of the cell which is finite. Dimensions of all cells are "even" and hence they represent semi-infinite homology classes of $\widetilde{L_{\text {an }}}(P)$. Note that this cellular decomposition is related to singular points of our Morse function.

The decomposition of $\widetilde{L_{\mathrm{an}}}(P)$ into Schubert cells gives the decomposition of $X_{p}$ into Schubert cells, which are connected with Wakimoto modules. It leads to the semi-infinite analogue of Cousin-Grothendieck resolution which gives two-sided BGG resolution.

\subsection{Morse Function}

As is well-known, $F_{p}=G / P=\mathscr{U} / T_{p}$ (where $\mathscr{U}$ is a compact form of $G, T_{p}$ is a compact form of $P$ ) is a symplectic manifold an orbit of the coadjoint representation of $\mathscr{U}$. Denote the symplectic form on $F_{p}$ by $\omega_{p}$. Let $\tau \in t_{p}$ (Lie algebra of $T_{p}$ ) be a regular element of $t_{p}$ and $h_{\tau}(\kappa)$ be the hamiltonian of the vector field $\tau$ on $F_{p}$. It is a Morse function on $F_{p}$ with singular points-images of the elements $\tilde{s} \in S(G) / S\left(G_{p}\right)$ in $F_{p}[8]$.

Let us define the semi-infinite Morse function $H_{\tau}(\gamma): \widetilde{L(P)} \rightarrow \mathbb{C}$. For any $\gamma: D^{2}$ $\rightarrow F_{p}, \gamma \in \widetilde{L(P)}$ we put

$$
H_{\tau}(\gamma)=\int_{\gamma} \omega_{p}-\int_{\partial \gamma} h_{\tau} d \varphi
$$

We call $H_{\tau}(\gamma)$ the Conley-Zehnder function [11].

Its singular points are such maps $\gamma: D^{2} \rightarrow F_{p}$, which transfer the border of $D^{2}$ to $\tilde{s} \in F_{p}$ (and winds the disk $D^{2}$ on a certain element of $\left.H_{2}\left(F_{p} ; \mathbb{Z}\right) \simeq \Lambda_{G} / \Lambda_{G_{p}}\right)$. So singular points of $H_{\tau}(\gamma)$ are labelled by $S_{\text {aff }}(G) / S_{\text {aff }}\left(G_{p}\right)$.

Let us compute the relative indices of singular points of $H_{\tau}(\gamma)$.

At first, recall how to compute the indices of singular points of $h_{\tau}(\kappa)$. Let $\Delta_{p}=\Delta(G) \backslash \Delta\left(G_{p}\right), \Delta_{p}=\Delta_{p}^{+} \cup \Delta_{p}^{-}, \Delta_{p}^{ \pm}=\Delta_{p} \cap \Delta_{ \pm}$. We put

$$
l_{p}(\tilde{s})=\#\left\{\alpha \in \Delta_{p}^{+}: \tilde{s} \alpha \in \Delta(G)_{-}\right\}
$$

(this definition is correct because $s\left(\Delta_{p}^{+}\right)=\Delta_{p}^{+}$, if $s \in S\left(G_{p}\right)$ ). The index of the point $\tilde{s}$ is equal to ind $(\tilde{\mathbf{S}})=2 l_{p}(\tilde{\mathbf{s}})$.

Denote $\hat{\Delta}_{p}=\widehat{\Delta}(G) \backslash \widehat{\Delta}\left(G_{p}\right)$. For $\tilde{s} \in S_{\text {aff }}(G) / S_{\text {aff }}\left(G_{p}\right)$, let

$$
\begin{aligned}
\widetilde{I t}_{p}(\tilde{s})= & \#\left\{\alpha \in \tilde{\Delta}_{p}^{+}: \alpha=l \delta+\beta, \beta \in \Delta_{p}^{+} ; \tilde{s} \alpha \in \widehat{\Delta}(G)_{-}\right\} \\
& -\#\left\{\alpha \in \hat{\Delta}_{p}^{+}: \alpha=l \delta+\beta, \beta \in \Delta_{p}^{-} ; \tilde{s} \alpha \in \widehat{\Delta}(G)_{-}\right\} .
\end{aligned}
$$

This definition is correct, because for any $b \in \Lambda_{G_{p}}$ and $\alpha \in \Delta_{p}^{+}$there is $\alpha^{\prime} \in \Delta_{p}^{-}:\left(\alpha^{\prime}, b\right)$ $=(\alpha, b)$. 
Now if we put ind(1) $=0$, then

$$
\operatorname{ind}(\tilde{s})=\widetilde{2 l t_{p}(\widetilde{s})}, \quad \tilde{s} \in S_{\text {aff }}(G) / S_{\text {aff }}\left(G_{p}\right) .
$$

So semi-infinite homologies of $\widetilde{L(P)}$ are contained in even dimensions $2 i$ and generated over $\mathbb{C}$ by elements of the set $M_{p}(i)$, where $M_{p}(i)=\left\{\tilde{s} \in S_{\mathrm{aff}}(G) / S_{\mathrm{aff}}\left(G_{p}\right)\right.$ : $\left.\overline{l t}_{p}(\tilde{s})=i\right\}$.

\subsection{Cellular Decomposition}

The cells of $\widetilde{L_{a n}}(P)$ are the orbits of the group $\hat{N}_{+}$. Let $\overline{\mathscr{U}}_{\bar{s}}=\hat{N}_{+} \cdot \tilde{s}$, $\tilde{s} \in S_{\text {aff }}(G) / S_{\text {aff }}\left(G_{p}\right), \tilde{s}$ is embedded onto $\widetilde{L_{\text {an }}}(P)$. Then $\overline{\mathscr{U}}_{\bar{s}}$ is identified with $\hat{N}_{p} \cap \tilde{s} \hat{N}_{+} \tilde{S}^{-1}$, where $\hat{N}_{p}$ is the subgroup of $L_{\text {an }} G^{\Lambda}$, which corresponds to Lie algebra $r_{p} \otimes \mathbb{C}((t))$ [note that $\hat{N}_{p} \cap s \hat{N}_{+} s^{-1}=\widehat{N}_{p} \cap \widehat{N}_{+}, s \in S_{\text {aff }}\left(G_{p}\right)$ ]. So, putting $\operatorname{dim} \overline{\mathscr{U}}_{1}=0$ we obtain that relative dimension $\operatorname{dim} \overline{\mathscr{U}}_{\bar{s}}=2 \overline{l t}_{p}(\tilde{s})$. It agrees with Sect. 4.3. Note that all cells are of "equal size" and the closure of $\overline{\mathscr{U}}_{\bar{s}}$ consists of $\overline{\mathscr{U}}_{\bar{s}^{\prime}}$, such that $\operatorname{dim} \overline{\mathscr{U}}_{\bar{s}^{\prime}}<\operatorname{dim} \overline{\mathscr{U}}_{\bar{s}^{\text {. }}}$.

We also obtain the decomposition of $X_{p}^{\text {an }}$ into Schubert cells. Let $s \in X_{p}$ be the image of $s \in S_{\mathrm{aff}}(G)$ in $X_{p}^{\mathrm{an}}$. Then the orbits of $s$ under the action of $\hat{N}_{+}$give this decomposition. Denote $\mathscr{U}_{s}=\hat{N}_{+} \cdot s \subset X_{p}^{\text {an }}$. Evidently, $\mathscr{U}_{s}$ is the product of the appropriate cells of $\widetilde{L_{\text {an }}}(P)$ and $Y_{p}$. The relative dimension of $\mathscr{U}_{s}\left(\right.$ we put $\operatorname{dim} \mathscr{U}_{1}=0$ ) is equal to $2 \overline{t_{p}}(s)$, defined as follows. We have $\Delta(G)=\Delta(P) \cup \Delta^{p}$. Let

$$
\hat{\Delta}(P)=\{l \delta+\alpha, \alpha \in \Delta(P), l \in \mathbb{Z}\}, \quad \hat{\Delta}^{p}=\left\{l \delta+\alpha, \alpha \in \Delta^{p}, l \in \mathbb{Z}\right\} .
$$

Then

$$
\overline{l t_{p}}(s)=\#\left\{\alpha \in \widehat{\Delta}(P) \cap \widehat{\Delta}(G)_{+}: s \alpha \in \widehat{\Delta}(G)_{-}\right\}-\#\left\{\alpha \in \hat{\Delta}^{p} \cap \widehat{\Delta}(G)_{+}: s \alpha \in \hat{\Delta}(G)_{-}\right\} .
$$

In particular,

$$
\begin{aligned}
\overline{l_{b}}(s)= & \#\left\{\alpha \in \widehat{\Delta}(G)_{+}: \alpha=l \delta+\beta, \beta \in \Delta_{+}, s \alpha \in \widehat{\Delta}(G)_{-}\right\} \\
& -\#\left\{\alpha \in \widehat{\Delta}(G)_{+}: \alpha=l \delta+\beta, \beta \in \Delta_{-}, s \alpha \in \widehat{\Delta}(G)_{-}\right\},
\end{aligned}
$$

and if $s=s^{\prime} \cdot t_{\gamma}, s^{\prime} \in S(G), b \in \Lambda_{G}$, then $\overline{l t_{\gamma}}(s)=l\left(s^{\prime}\right)+(2 \varrho, \gamma)$. We define also $l t_{p}(s)$ $=\overline{t_{p}}\left(s^{-1}\right)$.

\subsection{Connection with Wakimoto Modules}

We suppose that in a semi-infinite case there are analogues of functors $U, V, Z$ of Sect. 2, 3, which establish the connection of Wakimoto modules $W_{\chi, p}$ with sheaves on $X_{p}$.

The Wakimoto module $W_{s * \chi, s p s^{-1}}$ corresponds to the space of "local semiinfinite cohomology" with support on the Schubert cell of the invertible sheaf $H_{\mathscr{U}_{s}}^{\infty / 2-\overline{l t}_{p}(s)}\left(X_{p}, \widetilde{\xi}_{\chi}\right)$, where $\overline{l t_{p}}(s)$ is the (complex) relative codimension of the cell $\mathscr{U}_{s}$. In the next section we will give algebraic construction of Wakimoto modules which is similar to finite-dimensional construction of 2.4 and clarifies the notion of local semi-infinite cohomology.

The constructible sheaf on $X_{p}$, corresponding to the $L g^{\Lambda}$-module $M$, has the stalk at the point $\kappa$, isomorphic to the complex of semi-infinite homology (more exactly, its $w * \chi$ eigenvalue component with respect to the action of $\bar{h}$ ) 
$C_{\infty / 2+*}^{w * \chi}\left(a_{p, \kappa}^{*}, M\right)$, where $a_{p, \kappa}^{*}=\kappa a_{p}^{*} \kappa^{-1}=a_{p}^{*}$ is the Lie algebra of the stabilizer of $\kappa \in X_{p}$ and we take its decomposition

$$
a_{p, \kappa}^{*}=a_{p, \kappa}^{*+} \oplus a_{p, \kappa}^{*-}, \quad a_{p, \kappa}^{* \pm}=\kappa\left(a_{p, \kappa}^{*} \cap \hat{n}_{ \pm}\right) \kappa^{-1} .
$$

So, the Wakimoto module $W_{(s w) * \chi, s p s^{-1}}$ corresponds to the constant sheaf on the Schubert cell $\mathscr{U}_{s}$. The irreducible representation with dominant integral highest weight $L_{\chi}$ corresponds to the constant sheaf on $X_{p}$. Irreducible representations $L_{s * \chi}$ correspond to semi-infinite analogues of Goresky-MacPherson sheaves [4].

\subsection{Cousin-Grothendieck Resolution}

There is a semi-infinite analogue of Cousin-Grothendieck resolution $R_{p}^{\infty / 2+*}(\chi)$ of invertible sheaf $\tilde{\xi}_{\chi}$ with respect to Schubert stratification of $X_{p}$. Its terms are local cohomologies of $\widetilde{\xi}_{\chi}$ with support on the Schubert cell, that is Wakimoto modules:

$$
R_{p}^{\infty / 2+i}(\chi)=\underset{\substack{\operatorname{codim} \mathcal{U}_{s}=i \\ s \in S_{\mathrm{aff}}}}{\oplus} H_{\mathscr{U}_{s}}^{\infty / 2+i}\left(X_{p}, \tilde{\xi}_{\chi}\right) \simeq \underset{\substack{s \in S_{\text {aff }} \\ l t_{p}(s)=-i}}{\bigoplus} W_{s * \chi, s p s^{-1}}
$$

The cohomologies of this complex coincide with cohomologies of $\tilde{\xi}_{\chi}$ :

$$
H^{\infty / 2+i}\left(X_{p}, \tilde{\xi}_{\chi}\right)=0, \quad i \neq 0, \quad H^{\infty / 2}\left(X_{p}, \tilde{\xi}_{\chi}\right) \simeq L_{\chi} .
$$

[analogously, $H^{\infty / 2+i}\left(X_{p}, \widetilde{\xi}_{s * \chi}\right)=0$, if $\left.i \neq \overline{l t_{p}}(s), H^{\infty / 2+l t_{p}(s)}\left(X_{p}, \widetilde{\xi}_{s * \chi}\right) \simeq L_{\chi}\right]$. This is the analogue of the Borel-Weil-Bott theorem.

So $R_{p}^{\infty / 2+*}(\chi)$ is the resolution of an irreducible representation. It is the limit resolution of twisted resolutions on the usual flag manifold $X$. We call it two-sided BGG resolution. This is an analogue of generalized strong BGG resolution.

In Sect. 6 we will prove a similar two-sided BGG resolution in a different way. It is an analogue of generalized weak BGG resolution.

\subsection{Grassmanian Model}

There is a Grassmanian model of $\widetilde{L(P)}$ similar to the Grassmanian model of the usual flag manifold [40]. We give this model for $L_{\mathrm{pol}} S L_{2}$ [40], the other models for other algebras are direct generalizations of this.

Let $\mathscr{H}=L^{2}\left(S^{1}, \mathbb{C}^{2}\right)$ be Hilbert space. Let $e_{1}$ and $e_{2}$ be basic vectors in $\mathbb{C}^{2}$. We choose the basis $u_{i}, i \in \mathbb{Z}$ in $\mathscr{H}$, such that $u_{2 i}=e_{1} z^{i}, u_{2 i}=l_{2} z^{i}$. The shift operator $z$ transforms $u_{i}$ to $u_{2 i+1}$.

There is decomposition of $\mathscr{H}$ into a direct sum of mutually orthogonal subspaces:

$$
\mathscr{H}=H_{+} \oplus H_{-}^{(1)} \oplus H_{-}^{(2)},
$$

where $H_{+}$is generated (over $\mathbb{C}$ ) by $u_{i}, i>0, H_{-}^{(1)}-$ by $u_{2 i}, i \leqq 0, H_{-}^{(2)}-$ by $u_{2 i-1}, i \leqq 0$.

Let us consider the manifold $G r$ of all subspaces $H$ of $\mathscr{H}$, commensurable with $H_{-}^{(1)}$ or $H_{-}^{(2)}$ (see [40]), characterized by the property:

$z H C H$ and $z^{n-1} H / z^{n} H$ is one-dimensional. 
The manifold $G r$ is isomorphic to $\widetilde{L(B)}$ pol of $L s l_{2}$. The group $L_{\mathrm{pol}} S L_{2}$ acts on $G r$ naturally, the stabilizer of $H_{-}^{(1)}$ being the group $L_{\mathrm{pol}} B_{0}$ (the connected component of the unit of $L_{\mathrm{pol}} B$ ).

We say that an element $u \in \mathscr{H}$ is of finite order $q$ if $u=\sum_{i \geqq q} c_{i} u_{i}, c_{i} \in \mathbb{C}$. For $H \subset \mathscr{H}$ we put $Q_{H}=\{q \in \mathbb{Z}: H$ contains an element of order $q\}$. For the set of integers $Q$ we put $\sum_{Q}=\left\{H \in G r: Q_{H}=Q\right\}$.

The affine Weyl group $S_{\text {aff }}\left(S L_{2}\right)$ is freely generated by $s_{0}$ and $s_{1}$. Let us denote

$$
\begin{aligned}
& s_{(i)}=\underbrace{s_{0 \text { or } 1} \ldots s_{1} s_{0} s_{1}}_{-i \text { times }}, \quad i \leqq 0, \\
& s_{(i)}=\underbrace{s_{0 \text { or } 1} \ldots s_{0} s_{1} s_{0}}_{i \text { times }}, \quad i>0 .
\end{aligned}
$$

Then $\bar{u}_{s(r)}-$ the cell of $\overline{L(B)}$ coincides with $\sum_{Q_{i}}$, where

$$
Q_{i}=\{i, i-2, i-4, \ldots\} \text {. }
$$

It is the orbit of $H_{Q_{i}}=s_{(i)}$ under the action of $\hat{N}_{+}$.

\section{Wakimoto Modules: Construction}

In this section we will construct Wakimoto modules algebraically as certain modules over the algebra of infinitesimal automorphisms of the bundle over the formal neighbourhood of the cell of the manifold $\widetilde{L(p)}$. This is the generalization of the finite-dimensional construction of twisted Verma modules 2.4. But in the infinite-dimensional case we meet with some homological problems.

\subsection{The Case of Borel Subalgebra}

We start with the most important case $p=b$. Denote $W_{\chi}=W_{\chi, b}$. Wakimoto modules $W_{\chi}$ are boson representations of $L g^{\Lambda}$, which are interesting in conformal field theory. It is possible to obtain explicit formulae for these representations. The formulae for $L s l_{n}^{\Lambda}$ are represented in Sect. 7.

Consider the formal neighbourhood $\mathscr{N}$ of the cell $\overline{\mathscr{U}}_{1}$ of $\widetilde{L(B)}$. It is isomorphic to the linear space $N_{-} \otimes \mathbb{C}((t)) \simeq \mathbb{C}^{d(g)}((t)), d(g)=(\operatorname{dim} g-n) / 2$.

First of all let us study Lie algebra of vector fields on $\mathcal{N}$.

Let $\bar{\Gamma}$ be Heisenberg algebra with generators $a_{\alpha}(m), a_{\alpha}^{*}(m), \alpha \in \Delta_{-}, m \in \mathbb{Z}$ and commutation relations

$$
\left[a_{\alpha}(m), a_{\beta}(l)\right]=\left[a_{\alpha}^{*}(m), a_{\beta}^{*}(l)\right]=0, \quad\left[a_{\alpha}(m), a_{\beta}^{*}(l)\right]=\delta_{\alpha, \beta} \delta_{m,-l} .
$$

Let $\bar{M}$ be an irreducible representation of $\bar{\Gamma}$ with vacuum vector, annihilated by $a_{\alpha}(m), m>0, \alpha \in \Delta_{-}, a_{\alpha}^{*}(m), m \geqq 0, \alpha \in \Delta_{-}$. Introduce grading on $\bar{\Gamma}$ and $\bar{M}$, putting $\operatorname{deg} a_{\alpha}^{*}(m)=1, \operatorname{deg} a_{\alpha}(m)=-1$. Denote by $A_{0}$ the algebra of operators $\bar{M} \rightarrow \bar{M}$, commuting with all $a_{\alpha}^{*}(m)$ and by $A_{1}$ its normalizer in End $\bar{M}$. The space $A_{1} / A_{0}=W$ $=\underset{i \geq-1}{\oplus} W_{i}$ is a graded Lie algebra. 
It is easy to see that $W_{1}$ consists of the operators $\sum_{\alpha \in \Delta} \varrho_{\alpha}(m) a_{\alpha}(m)$, where $\varrho_{\alpha}(m) \in \mathbb{C}, l \in \mathbb{Z}$. So $W_{-1}$ is identified with $\mathscr{N}$. Denote $\mathcal{N}_{+}=N_{-}^{m \geq l^{\prime}} \otimes t \mathbb{C}[[t]]$. We have

Proposition 2 [18]. 1. The Lie algebra $W_{0}$ is isomorphic to the algebra of operators $a: \mathscr{N} \rightarrow \mathscr{N}$, such that $\operatorname{dim}\left(\left(a\left(\mathscr{N}_{+}\right)+\mathscr{N}_{+}\right) / \mathscr{N}_{+}\right)<\infty ; W_{0} \oplus W_{-1}$ is normalizer of $W_{-1}$ in $W$;

2. The Lie algebra $W$ is Cartan prolongation of the pair $\left(W_{0}, W_{-1}\right) . W$ is identified with the Lie algebra of vector fields on $\mathcal{N}$.

It is interesting to compute the cohomology of $W$ with coefficients in $A_{0}$, which is identified with the space of the functions on $\mathscr{N}$.

We can change $W$ by the Lie algebra $W_{\infty}$ of "finite" vector fields, which is the Cartan prolongation of the pair $\left(g l_{\infty}, V_{\infty}\right)$, where $g l_{\infty}$ is the injective limit of $g l_{n}$, and $V_{\infty}$ is the injective limit of vector representation $V_{n}$ of $g l_{n}$. Denote by $F\left(V_{\infty}\right)$ the space of functions on $V_{\infty}$ and consider $H^{*}\left(W_{\infty}, F\left(V_{\infty}\right)\right)$. $W_{\infty}=W_{\infty,-1} \oplus \tilde{W}_{\infty}$, where $\tilde{W}_{\infty} \simeq \underset{i \geqq 0}{\oplus} W_{\infty, i}$. As is well-known,

$$
\begin{gathered}
H^{*}\left(W_{\infty}, F\left(V_{\infty}\right)\right) \simeq H^{*}\left(\widetilde{W}_{\infty}, \mathbb{C}\right) \simeq H^{*}\left(g l_{\infty}, \mathbb{C}\right) \simeq \Lambda^{*}\left(e_{1}, e_{2}, \ldots\right), \\
\operatorname{deg} e_{i}=2 i-1 \quad, i=1,2, \ldots
\end{gathered}
$$

Here the first isomorphism is given by the Schapiro lemma and the second is proved in [25].

We obtain the analogous result for $W$.

Theorem 1. $H^{*}\left(W, A_{0}\right) \simeq H^{*}\left(W_{0}, \mathbb{C}\right)$.

Proof. The isomorphism $H^{*}\left(W, A_{0}\right) \simeq H^{*}\left(\underset{i \geqq 0}{\oplus} W_{i}, \mathbb{C}\right)$ is given by the Schapiro
lemma and the proof of the isomorphism

is the same as in [25].

$$
H^{*}\left(\underset{i \geqq 0}{\oplus} W_{i}, \mathbb{C}\right) \simeq H^{*}\left(W_{0}, \mathbb{C}\right)
$$

The cohomology ring $H^{*}\left(W_{0}, \mathbb{C}\right)$ was computed in [22].

$$
H^{*}\left(W_{0}, \mathbb{C}\right) \simeq S^{*}\left(c_{1}, c_{2}, \ldots\right), \quad \operatorname{deg} c_{i}=2 i, i=1,2, \ldots .
$$

Note that $H^{2}\left(W_{0}, \mathbb{C}\right) \simeq \mathbb{C}$ is generated by the well-known Tate [42] or "Japanese" [12] or "wedge" [31a] cocycle $c_{1}$ and so $H^{2}\left(W, A_{0}\right) \simeq \mathbb{C}$. We can give the description of the cocycle $\tilde{c}_{1}$ generating $H^{2}\left(W, A_{0}\right)$. Let $W_{1}$ and $W_{2}$ be two vector fields on $\mathscr{N}$ from $W$. Any point $\kappa \in \mathscr{N}$ determines two elements $\bar{w}_{1}(\kappa)$ and $\bar{w}_{2}(\kappa)$ from $W_{0}$, which are linearizations of $W_{1}$ and $w_{2}$ at $\kappa$. We put

$$
\left[\tilde{c}_{1}\left(w_{1}, w_{2}\right)\right](\kappa)=c_{1}\left(\bar{w}_{1}(\kappa), \bar{w}_{2}(\kappa)\right) .
$$

Now we pass to $L g^{\Lambda}$. It is clear that $L g$ acts on $\mathcal{N}$ and it gives the embedding $\operatorname{Lg} \hookrightarrow W$.

Proposition 3 [18]. Composition

applied to $\tilde{c}_{1}$ is 0.

$$
H^{2}\left(W, A_{0}\right) \rightarrow H^{2}\left(L g, A_{0}\right) \rightarrow H^{2}\left(L g, A_{0} / \mathbb{C}\right),
$$


Proof. Short exact sequence $0 \rightarrow \mathbb{C} \rightarrow A_{0} \rightarrow A_{0} / \mathbb{C} \rightarrow 0$ gives

$$
H^{2}(L g, \mathbb{C}) \stackrel{\varepsilon_{1}}{\longrightarrow} H^{2}\left(L g, A_{0}\right) \stackrel{\varepsilon_{2}}{\longrightarrow} H^{2}\left(L g, A_{0} / \mathbb{C}\right) .
$$

According to Schapiro lemma,

$$
H^{2}\left(L g, A_{0}\right) \simeq H^{2}\left(L b_{+}, \mathbb{C}\right) \simeq H^{2}(L h, \mathbb{C}) .
$$

Denote the image of the projection of $\tilde{c}_{1}$ onto $H^{2}\left(\operatorname{Lg}, A_{0}\right)$ by $\bar{c}_{1}$, and the corresponding element of $H^{2}(L h, \mathbb{C})$ by $\bar{c}$. We should show that $\bar{c}$ lies in the image of $\varepsilon_{1}$. Recall that $H^{2}(L g, \mathbb{C}) \simeq \mathbb{C}$ is generated by the central charge. It means that we should show that $\bar{c}$ (central extension of $L h$ ) is the restriction of the central extension of $\mathrm{Lg}$. It follows from the computations below.

Let us compute $\bar{c}$. In order to do it we should express operators $H_{\alpha}(m) \in \operatorname{Lg}^{\Lambda}\left(H_{\alpha}\right.$ is the coroot, dual to the root $\alpha \in \Delta)$ via operators $a_{\beta}(m), a_{\beta}^{*}(m)$.

We need some preparations. For any countable set $A(m), m \in \mathbb{Z}$, of operators we put $A(z)=\sum_{m \in \mathbb{Z}} A(m) z^{m}, \dot{A}(z)=z \frac{d}{d z} A(z)$. Introduce normal ordering ::, as usual [19].

Operators $H_{\alpha}(m)$ act on $\bar{M}$ as follows:

$$
H_{\alpha}(z)=\sum_{\beta \in \Delta_{-}}(\bar{\alpha}, \beta): a_{\beta}(z) a_{\beta}^{*}(z): .
$$

Using the Wick theorem we obtain:

$$
\begin{aligned}
{\left[H_{\alpha}(m), H_{\beta}(l)\right] } & =-\delta_{m,-l} \cdot m \sum_{\gamma \in \Delta_{+}}(\bar{\alpha}, \gamma)(\bar{\beta}, \gamma) \\
& =\delta_{m,-l} \cdot m(\bar{\alpha}, \bar{\beta})\left(-c_{g}\right),
\end{aligned}
$$

where $c_{g}$ is dual Coxeter number of $g$ [29]. We see that $\bar{c}$ is the restriction of the central extension of $L g$ and Proposition 3 follows.

We obtain the following result.

Theorem 2 [18]. The embedding $L g \hookrightarrow W$ is lifted to the embedding $L^{\Lambda} \hookrightarrow A_{1}$. The set of these embeddings is the principal homogeneous bundle over $H^{1}\left(\mathrm{Lg}, A_{0}\right)$. There is natural homomorphism $h^{*} \rightarrow H^{1}\left(L g, A_{0}\right)$. There is an n-parameter family of $\mathrm{Lg}^{\Lambda}$-modules $\bar{W}_{\bar{\chi}}, \bar{\chi} \in h^{*}$ with central charge $-c_{g}$ in $\bar{M}$.

We call $\bar{W}_{\bar{\chi}}$ a restricted Wakimoto module. It is characterized by the homological property:

$$
H^{\infty / 2}\left(r_{p} \otimes \mathbb{C}((t)), \bar{W}_{\bar{\chi}}\right) \simeq \mathbb{C}_{\bar{\chi}} ; \quad H^{\infty / 2+i}\left(r_{p} \otimes \mathbb{C}((t)), \bar{W}_{\bar{\chi}}\right)=0, \quad i \neq 0 .
$$

$\bar{W}_{\bar{\chi}}$ corresponds to the cell of $\widetilde{L(B)}$. We studied $\bar{W}_{\bar{\chi}}$ in [19] (see also Appendix B).

In order to construct Wakimoto modules $W_{\chi}$ we should consider the fibering over $\mathscr{N}$ with the fiber $\pi_{k^{\prime}}$-Fock representation of $L h^{\Lambda}$ with vacuum vector annihilated by $h \otimes \mathbb{C}[[t]]$ and central charge $k^{\prime}$.

So we should consider the extension $\Gamma$ of $\bar{\Gamma}$ by generators $b_{i}(m), i=1, \ldots, n$, $m \in \mathbb{Z}$, commuting with $\Gamma$ and with commutation relations $\left[b_{i}(m), b_{j}(l)\right]$ $=\delta_{m,-l}\left\langle H_{i}, H_{j}\right\rangle$. The subalgebra of $\Gamma$ is identified with the algebra of infinitesimal automorphisms of our fibering. So $L g^{\Lambda}$ imbeds into $\Gamma$ and it equips $\bar{W}_{\bar{\chi}} \otimes \pi_{k^{\prime}}$ with the structure of an $L g^{\Lambda}$-module with central charge $k=k^{\prime}-c_{g}$ and highest weight $\chi=(\bar{\chi}, k)$. So we obtain an $(n+1)$-parameter family of $L g^{\Lambda}$-modules, which is the family of Wakimoto modules $W_{\chi}[18]$. 


\subsection{General Case}

Now we will construct $W_{\chi, p}$ with an arbitrary parabolic subalgebra $p$ of $g$.

We introduce some notations. The subgroup $V_{p}$ of $G$ is the product of semisimple subgroup: $G_{p}=G_{p}^{(1)} \cdot \ldots \cdot G_{p}^{(q)}$ (where $G_{p}^{(i)}$ are simple subgroups of $G_{p}$ ) and of the abelian subgroup $H_{p}$. We assume that corresponding Lie algebras $g_{p}^{(i)}$, $i=1, \ldots, q$ and $h_{p}$ are mutually orthogonal with respect to the Killing form.

Let $M_{\left(\chi, \mathbf{k}_{p}\right)}^{p^{*}}$ be the contragradient Verma module over $L g_{p}^{A}$ :

$$
M_{\left(\chi, \mathbf{k}_{p}\right)}^{p^{*}}=M_{\left(\chi_{1}, k_{p}^{(1)}\right)}^{*} \otimes \ldots \otimes M_{\left(\chi_{q}, k_{p}^{(q)}\right)}^{*},
$$

where $M_{\left(\chi_{i}, k_{p}^{(i)}\right)}^{*}$ is a contragradient Verma module over $L g_{p}^{(i) \Lambda}$. Let $\pi_{\left(\chi^{\prime}, k_{p}^{\prime}\right)}$ be a Fock representation of $L h_{p}^{\Lambda}$ with central charge $k_{p}^{\prime}$ and highest weight $\chi^{\prime}$.

Consider the fibering over the formal neighbourhood $\mathscr{N}_{p}$ of the cell $\overline{\mathscr{U}}_{1} \subset \widetilde{L(P)}$ with fiber $M_{\left(\chi, \mathbf{k}_{p}\right)}^{p^{*}} \otimes \pi_{\left(\chi^{\prime}, k_{p}^{\prime}\right)}=M_{\chi, \chi^{\prime}, \mathbf{k}_{p}, k_{p}^{\prime}}^{p}$

We define the algebra $W^{p}$ of vector fields on the space $\mathcal{N}_{p}$ [which is isomorphic to $\left.R_{p} \otimes \mathbb{C}((t))\right]$ in the same way as in 5.1. Let $\Gamma_{p}$ be a Heisenberg algebra with generators $a_{\alpha}(m), a_{\alpha}^{*}(m), \alpha \in \Delta^{p}, m \in \mathbb{Z}$ and commutation relations (1). Let $\bar{M}_{p}$ be its irreducible representation with a vacuum vector, annihilated by $a_{\alpha}(m), \alpha \in \Delta^{p}$, $m>0$ and $a_{\alpha}^{*}(m), \alpha \in \Delta^{p}, m \geqq 0$. Denote by $A_{0}^{p}$ the algebra of operators $\bar{M}_{p} \rightarrow \bar{M}_{p}$, commuting with $a_{\alpha}^{*}(m), \alpha \in \Delta^{p}, m \in \mathbb{Z}$, and by $A_{1}^{p}$ its normalizer $W^{p}=A_{1}^{p} / A_{0}^{p}$ is identified with a Lie algebra of vector fields in End $\bar{M}_{p}$ on $\mathscr{N}_{p}$.

The algebra $\mathrm{Aut}_{p}$ of infinitesimal automorphisms of the fibering defined above is a semi-direct sum of $W^{p}$ and of the algebra

$$
\text { End } M_{\chi, \chi^{\prime}, \mathbf{k}_{p}, k_{p}^{\prime}}^{p} \otimes A_{0}^{p}=\text { End }_{p} .
$$

The extension of $W^{p}$ by $A_{0}^{p}$, defined in 5.1 gives the extension of $W^{p}$ by End

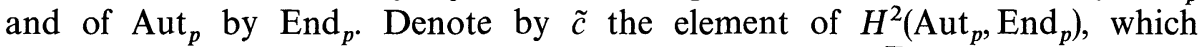
corresponds to the representation of $\mathrm{Aut}_{p}$ in $M_{\chi, \chi^{\prime}, \mathbf{k}_{p}, k_{p}^{\prime}}^{p} \otimes \bar{M}_{p}$.

We want to obtain the condition, when $L g^{\Lambda}$ may be embedded into Aut ${ }_{p} . L g$ acts on $\mathscr{N}_{p}$ and hence imbeds into $W^{p}$. So $L g^{\Lambda}$ imbeds into Aut ${ }_{p}$ if and only if the composition

$$
H^{2}\left(\operatorname{Aut}_{p}, \operatorname{End}_{p}\right) \rightarrow H^{2}\left(L g, \operatorname{End}_{p}\right) \rightarrow H^{2}\left(L g, \operatorname{End}_{p} / \mathbb{C}\right),
$$

applied to $\tilde{c}$ gives 0 .

Proposition 4. The composition

$$
H^{2}\left(\operatorname{Aut}_{p}, \operatorname{End}_{p}\right) \rightarrow H^{2}\left(L g, \operatorname{End}_{p}\right) \rightarrow H^{2}\left(L g, \operatorname{End}_{p} / \mathbb{C}\right),
$$

applied to $\tilde{c}$, gives 0 , if and only if the following conditions are satisfied:

$$
k_{p}^{(i)}=k_{p}^{\prime}-c_{g_{p}^{(\imath)}} .
$$

Proof. The short exact sequence $0 \rightarrow \mathbb{C} \rightarrow \operatorname{End}_{p} \rightarrow \operatorname{End}_{p} / \mathbb{C} \rightarrow 0$ gives:

$$
H^{2}(L g, \mathbb{C}) \rightarrow H^{2}\left(L g, \operatorname{End}_{p}\right) \rightarrow H^{2}\left(L g, \operatorname{End}_{p} / \mathbb{C}\right) .
$$

As in Proposition 3, we see that, according to the Schapiro lemma,

$$
H^{2}\left(L g, \operatorname{End}_{p}\right) \simeq H^{2}\left(L_{p}, M_{\chi, \chi^{\prime}, \mathbf{k}_{p}, k_{p}}^{p}\right)=\bigoplus_{i=1}^{q} H^{2}\left(L g_{p}^{(i)}, M_{\left(\chi_{i}, k_{p}^{(i)}\right)}^{*}\right) \oplus H^{2}\left(L h_{p}, \pi_{\chi^{\prime}, k_{p}^{\prime}}\right),
$$


and we should show that the restriction of $\tilde{c}$ to the latter cohomology space is the restriction of the central charge of $L g$. In order to do it we should compute the extension on $h \subset p$.

For any $H_{\beta} \in h$ we have $H_{\beta}=H_{\beta}^{p}+\sum_{i=1}^{q} H_{\beta, i}$, where $H_{\beta}^{p} \in h_{p}$ and $H_{\beta, i} \in g_{p}^{(i)}$.

We have the action of $H_{\beta}(m)$ in $M_{\chi, \chi^{\prime}, \mathbf{k}_{p}, k_{p}^{\prime}}^{*} \otimes \bar{M}_{p}$ :

$$
H_{\beta}^{(z)}=\sum_{\alpha \in \Delta^{p}}: a_{\alpha}(z) a_{\alpha}^{*}(z):(\alpha, \bar{\beta})+\bar{H}_{\beta}^{p}(z)+\bar{H}_{\beta, i}(z),
$$

where $H_{\beta}^{p}(m)$ denotes the action of $H_{\beta}^{p}(m)$ in $\pi_{\chi^{\prime}, k_{r}}, \bar{H}_{\beta, i}(m)$ denotes the action of $H_{\beta, i}(m)$ in $M_{\chi_{2}, k_{p}^{(2)}}^{*}$.

We have:

$$
\left[H_{\beta}(m), H_{\gamma}(l)\right]=m \delta_{m,-l}\left(-\sum_{\alpha \in \Delta^{p}}(\alpha, \bar{\beta})(\alpha, \bar{\gamma})+\sum_{i=1}^{q} k_{p}^{(i)}\left\langle H_{\beta, i}, H_{\gamma, i}\right\rangle+k_{p}^{\prime}\left\langle H_{\beta}^{p}, H_{\gamma}^{p}\right\rangle\right) .
$$

Evidently, $\left\langle H_{\beta}, H_{\gamma}\right\rangle=\sum_{i=1}^{q}\left\langle H_{\beta, i}, H_{\gamma, i}\right\rangle+\left\langle H_{\beta}^{p}, H_{\gamma}^{p}\right\rangle$. We must obtain

$$
\left[H_{\beta}(m), H_{\gamma}(l)\right]=m \delta_{m,-l}(\bar{\beta}, \bar{\gamma}) \cdot k .
$$

So we see that the following conditions must be satisfied for it:

$$
k_{p}^{(i)}=k_{p}^{\prime}-c_{g_{p}^{(l)}},
$$

(where $c_{g_{p}^{(t)}}$ denotes dual Coxeter number of $g_{p}^{(i)}$ ).

Then (2) is (3) with $k=k_{p}^{\prime}-c_{g}$.

The proposition is proved.

So we obtain that if (4) is satisfied, then $M_{\chi, \chi^{\prime}, \mathbf{k}_{p}, k_{p}}^{p} \otimes \bar{M}_{p}$ is equipped with the structure of an $\mathrm{Lg}^{\Lambda}$-module, which is isomorphic to $W_{\chi, p}$, where $\chi=\left(\chi, \chi^{\prime}, k\right)$, with central charge $k=k_{p}^{\prime}-c_{g}$.

Our construction shows that $W_{\chi, p}$ is the $L g^{\Lambda}$-module, "semi-infinitely induced" from the $\left(L g_{p} \oplus L h_{p}\right)^{\Lambda}$-module $M_{\chi, \chi^{\prime}, \mathbf{k}_{p}, k_{p}}^{p}$ and the existence of a central extension imposes the constraints (4) on the central charges. Indeed, it is possible to induce from any representation of $\left(L g_{p} \oplus L h_{p}\right)^{\Lambda}$, if the conditions (4) are satisfied.

In particular, if we induce from a Verma module, then we obtain the module $W_{\chi, p^{*}}^{*}$ corresponding to the cell of $X_{p^{*}}^{+}$.

Our construction also says that it is possible to put the sheaf of $\left(L g_{p} \oplus L h_{p}\right)^{\Lambda}$ modules on $\widetilde{L(p)}$ into correspondence with highest weight $L^{\Lambda}$-module, by taking the complex of semi-infinite homology $C_{\infty / 2+*}\left(r_{p} \otimes \mathbb{C}((t)), M\right)$ as the stalk of this sheaf. The corresponding sheaves may be lifted (in some sense) to the constructible sheaves on $X_{p}$ (see Sect. 4).

\section{Two-Sided Bernstein-Gelfand-Gelfand Resolutions}

In this section we construct and prove two-sided Bernstein-Gelfand-Gelfand (BGG) resolutions of irreducible module over $\mathrm{Lg}^{\Lambda}$, consisting of Wakimoto modules. The approach we develop is an alternative to that of Sect. 4, where similar resolutions appeared to be Cousin-Grothendieck on the semi-infinite flag manifold. 
Our resolutions are the subcomplexes of the semi-infinite de Rham complex in the neighbourhood of the big cell on the semi-infinite flag manifold (for a finitedimensional counterpart see 2.4).

Let $\Gamma_{p}^{+}$be the supersymmetric extension of $\Gamma_{p}$ by odd generators $\varphi_{\alpha}(m), \varphi_{\alpha}^{*}(m)$, $\alpha \in \Delta^{p}, m \in \mathbb{Z}$, commuting with $\Gamma$ with the following anticommutation relations:

$$
\begin{gathered}
{\left[\varphi_{\alpha}(m), \varphi_{\beta}(l)\right]_{+}=\left[\varphi_{\alpha}^{*}(m), \varphi_{\beta}^{*}(l)\right]_{+}=0,} \\
{\left[\varphi_{\alpha}(m), \varphi_{\beta}^{*}(l)\right]_{+}=\delta_{\alpha, \beta} \delta_{m,-l} .}
\end{gathered}
$$

Let $M_{p}^{+}$be the irreducible representation of $\Gamma_{p}^{+}$with vacuum vector annihilated by $a_{\alpha}(m), \varphi_{\alpha}(m), \alpha \in \Delta^{p}, m>0$ and by $a_{\alpha}^{*}(m), \varphi_{\alpha}^{*}(m), \alpha \in \Delta^{p}, m \geqq 0$. Introduce grading on $\Gamma_{p}^{+}$and $M_{p}^{+}$putting $\operatorname{deg} a_{\alpha}(m)=\operatorname{deg} \varphi_{\alpha}(m)=-1, \operatorname{deg} a_{\alpha}^{*}(m)=\operatorname{deg} \varphi_{\alpha}^{*}(m)=1$. Denote by $A_{0}^{p+}$ the algebra of operators $M_{p}^{+} \rightarrow M_{p}^{+}$, commuting with all $a_{\alpha}^{*}(m), \varphi_{\alpha}^{*}(m)$ and let $A_{1}^{p+}$ be the normalizer of $A_{0}^{p+}$ in the Lie superalgebra. The superalgebra $A_{1}^{p+} / A_{0}^{p+}$ $=W_{p}^{+}=\underset{i \geq-1}{\oplus} W_{p, i}^{+}$is a graded Lie superalgebra. We see that $W_{p,-1}^{+}$consists of the operators

$$
\sum_{\alpha \in \Delta^{P}, m \geqq l} \varrho_{\alpha}(m) a_{\alpha}(m)+\sum_{\alpha \in \Delta^{P}, m \geqq l^{\prime}} \varrho_{\alpha}^{\prime}(m) \varphi_{\alpha}(m), \quad \text { where } \quad l, l^{\prime} \in \mathbb{Z},
$$

and so $W_{p_{1}-1}^{+}$is identified with the tangent bundle $T^{+} \mathcal{N}_{p}$ over $\mathscr{N}_{p}$ [the formal neighbourhood of the cell $\left.\overline{\mathscr{U}}_{1} \subset \widetilde{L(p)}\right]$ with the changed parity of fibers. $W_{p}^{+}$is identified with Lie superalgebra of vector fields on $T^{+} \mathcal{N}_{p}$. In the same way as in Sect. 5, we obtain: $H^{2}\left(W_{p}^{+}, A_{0}^{p+}\right) \simeq \mathbb{C}$. The generator $\tilde{c}_{1}^{+}$of $H^{2}\left(W_{p}^{+}, A_{0}^{p+}\right)$ is induced by Tate-"Japanese" cocycle $c_{1}^{+}$from $H^{2}\left(W_{p, 0}^{+}, \mathbb{C}\right) \cong \mathbb{C}$.

Now change the grading on $\Gamma_{p}^{+}$and $M_{p}^{+}$, putting $\operatorname{deg}_{0} a_{\alpha}(m)=\operatorname{deg}_{0} \mathscr{A}_{\alpha}^{*}(m)=0$, $\operatorname{deg} \varphi_{\alpha}(m)=-1, \operatorname{deg} \varphi_{\alpha}^{*}(m)=1$. There is a canonical element $\tilde{d}$ (differential) in $\Gamma_{p}^{+}$ such that $\operatorname{deg}_{0} \tilde{d}=1,[\tilde{d}, \tilde{d}]_{+}=0$, which endows $M_{p}^{+}$with the structure of complex $R_{p}^{*}=\oplus_{i \in \mathbb{Z}} R_{p}^{i}$. It is evident that their cohomologies are contained in the $0^{\text {th }}$ dimension and equal to $\mathbb{C}$. This complex is the semi-infinite analogue of de Rham complex on $\mathscr{N}_{p}$.

On the other hand, let us consider the usual de Rham complex $\Omega^{*}$ on the big cell $X_{1}$ of the usual flag manifold $X\left(G_{p}\right)$ of the group $L G_{p}^{A}, \Omega_{p}^{*}=\underset{i \geqq 0}{\oplus} \Omega_{p}^{i}$. Denote by $F_{p}$ the space of the functions on $X_{1}$. The tensor product complex $\bar{R}_{p}^{*}=R_{p}^{*} \otimes \Omega_{p}^{*}$ $=\oplus_{i \in \mathbb{Z}} \bar{R}_{p}^{i}$ may be considered as the semi-infinite de Rham complex on the product of $X_{1}$ and $\mathscr{N}_{p}$. Denote by $d$ its differential. Note that the product of $\mathscr{N}_{p}$ and $X_{1}$ is isomorphic to the cell of the manifold $\bar{X}_{p}$ (see 4.2). Denote by $\widetilde{W}_{p}^{+}$the Lie superalgebra of vector fields on $T^{+}\left(\mathscr{N}_{p} \times X_{1}\right)$ (tangent bundle over $\mathscr{N}_{p} \times X_{1}$ with changed parity of fibers). The Lie algebra $L g$ acts on $T^{+}\left(\mathscr{N}_{p} \times X_{1}\right)$ and hence $L g$ imbeds into $\widetilde{W}_{p}^{+}$, the image commuting with $d$. We have the following

Proposition 5. The composition

$$
H^{2}\left(W_{p}^{+} ; A_{0}^{p+}\right) \rightarrow H^{2}\left(\tilde{W}_{p}^{+}, A_{0}^{p+} \otimes F_{p}\right) \rightarrow H^{2}\left(L g \oplus \mathbb{C} d, A_{0}^{p+} \otimes F_{p}\right)
$$

(the first mapping is due to the Schapiro lemma) transforms $\tilde{c}_{1}^{+}$to 0. 
Proof is analogous to the proof of Propositions 3 and 4 (we should consider the superconformal current algebra $\mathrm{Lg}^{+}$[32] which imbeds into $\widetilde{W}_{p}^{+}$, and furthermore is the same as in the proof of Propositions 3 and 4).

So $\bar{R}_{p}^{*}$ is equipped with the structure of complex of $L g^{\Lambda}$-modules. Its cohomologies are trivial in all dimensions except $0^{\text {th }}$, where they are equal to $\mathbb{C}$. Simple calculation by virtue of those of the proof of Propositions 3 and 4 show that the central charge of $\mathrm{Lg}^{A}$ is equal to 0 (due to supersymmetric "cancellation of anomalies").

Now let $L_{\chi}$ be an irreducible representation of $L g^{A}$ with dominant integral highest weight $\chi$. Consider the complex $\bar{R}_{p}^{*}(\chi)=\bar{R}_{p}^{*} \otimes L_{\chi}$. It is an $L g^{A}$-module with highest weight $\chi$. Hence, the Casimir element Cas of $L g^{A}[29]$ acts on $\bar{R}_{p}^{*}(\chi)$, commuting with differential $d$. So $\bar{R}_{p}^{*}(\chi)$ decomposes into the direct sum of subcomplexes $\bar{R}_{p}^{*}(\chi)=\oplus \bar{R}_{p}^{*}(\chi)_{\theta}$, where

$$
\bar{R}_{p}^{*}(\chi)_{\theta}=\left\{c \in \bar{R}_{p}^{*}(\chi) \mid \exists \mathrm{m}:(\mathrm{Cas}-\theta)^{m} c=0\right\} .
$$

Denote $R_{p}^{*}(\chi)=\bar{R}_{p}^{*}(\chi)_{(\chi+2 \varrho, \chi)}$. Cohomologies of this complex are contained in the $0^{\text {th }}$ dimension and are isomorphic to $L_{\chi}$.

$R_{p}^{*}(\chi)$ is a two-sided BGG resolution corresponding to parabolic subalgebra $p$ of $g$.

Theorem 3. $R_{p}^{i}(\chi)=\underset{\substack{s \in S_{\text {aff }} \\ l t_{p}(s)=-i}}{\oplus} W_{s * \chi, p}$.

Proof is equivalent to the standard [26]. It is easy to see that $R^{*}(\chi)$ is free over $a_{p}^{-}$ and the dual to $R^{*}(\chi)$ is free over $a_{p}^{+}$. So there is filtration of $R_{p}^{*}(\chi)$ by $L g^{A}$-modules, whose adjoint quotients are isomorphic to Wakimoto modules $W_{\chi^{\prime}, p}$. Highest weights $\chi^{\prime}$ of these modules lie on the set $\tilde{\chi}-\sum \gamma_{i}, \tilde{\chi} \in L_{\chi}$, where $\gamma_{i}$ are different roots of $\mathrm{Lg}^{\Lambda}$. Applying the arguments of [26] we see that the eigenvalue of Cas is equal to $(\chi+2 \varrho, \chi)$ only if

$$
\chi^{\prime}=s(\chi)-\sum_{i=1}^{l(s)} \gamma_{i}=s(\chi+\varrho)-\varrho=s * \chi,
$$

where $\left\{\gamma_{1}, \ldots, \gamma_{l(s)}\right\}=\left\{\gamma \in \widehat{\Delta}_{+}: s^{-1} \gamma \in \hat{\Delta}_{-}\right\}$. In $R_{p}^{*}(\chi)$ the corresponding module $W_{s * \chi, p}$ lies in the dimension $-l t_{p}(s)$. After all, the fact that filtration indeed splits follows from homological considerations in spirit of [41].

Our resolutions enable us to compute the semi-infinite cohomology of twisted parabolic subalgebras $a_{p}$ and $\left.r_{p} \otimes \mathbb{C}(t)\right)$ with coefficients in the irreducible representation (semi-infinite Borel-Weil-Bott theorem).

Theorem 4. 1. $H^{\infty / 2+i}\left(a_{p}, L_{\chi}\right)=\underset{\substack{s \in \text { Saff }_{l t_{p}(s)=-i} \\ \oplus}}{ } \mathbb{C}_{s^{*} \chi}$ as $\hat{h}$-module,

2. $H^{\infty / 2+i}\left(n_{+} \otimes \mathbb{C}\left((t), L_{\chi}\right)=\underset{\substack{s \in S_{\text {aff }} \\ l_{t+}(s)=-i}}{\bigoplus} \pi_{\left(s * \bar{x}, k+c_{g}\right)}\right.$ (as an Lhi-module).

Proof. The spectral sequence corresponding to our resolution degenerates in the first term and gives the result.

We use this result for computing the (co)homology of Lie algebra $r_{p} \otimes \mathbb{C}[[t]]$ of currents on the line to a nilpotent subalgebra.

We will formulate the result only for the algebra $n_{+} \otimes \mathbb{C}[t]$.

Denote $\bar{S}_{\text {aff }}=\Lambda_{+} \times S$, where $\Lambda_{+}=\bigoplus_{i=1}^{n} \mathbb{Z}_{+} \alpha_{i}$. For $s \in \bar{S}_{\text {aff }} l t_{b_{+}}(s)=l(s)$. 
Theorem 5. $H_{i}\left(n_{+} \otimes \mathbb{C}[t]\right) \simeq \underset{\substack{s \in \bar{S}_{\text {aff }} \\ l(s)=i}}{\bigoplus} \mathbb{C}\left[h_{j}(m)\right]_{m=1, \ldots, l_{j}(s)}^{j=1, \ldots, n}$, as $h \otimes \mathbb{C}[t]$ module, where $l_{j}(s), j=1, \ldots, n$ are defined as follows:

$$
\varrho-s(\varrho)=\sum_{j=1}^{n} l_{j}(s) \alpha_{j}, \quad l_{j}(s) \in \mathbb{Z}_{+}, h_{j} \text { are generators of } h .
$$

Proof is based on the study of the action of the homologies of $n_{+} \otimes \mathbb{C}[t]$ on its semiinfinite homology and follows from Theorem 4.

Detailed proof and related results will be published elsewhere.

Note that the standard methods of computing the (co)homologies of current algebras [17] fail in this case. Thus, Theorem 5 is one of the interesting applications of our theory.

\section{Examples}

\subsection{Explicit Formulae for $W_{\chi}$ over $L s l_{n+1}^{A}$}

We give the explicit formulae for the action of the generators of $L s l_{n+1}^{\Lambda}$ which were obtained in [18]. For the simplest case $L s l_{2}^{\Lambda}$ they were obtained by Wakimoto in [44] - that is why we call $W_{\chi}$ Wakimoto modules. Note also that formulas for $L s l_{2}^{A}$ and $L s l_{3}^{A}$ were independently obtained by A. B. Zamolodchikov (unpublished).

Denote by $E_{i}, H_{i}, F_{i}, i=1, \ldots, n$, standard generators of $s l_{n+1}$. Denote $a_{i j}(m)$ $=a_{-\alpha_{i}-\ldots-\alpha_{j}}(m), 1 \leqq i \leqq j \leqq n, a_{i j}^{*}(m)=a_{-\alpha_{i}-\ldots-\alpha_{j}}, 1 \leqq i \leqq j \leqq n$. Commutation relations of $a_{i j}(m), a_{i j}^{*}(m)$ are given in Sect. 5 .

Then

$$
\begin{aligned}
E_{i}(z)= & : a_{i i}^{*}\left(\sum_{j=1}^{i-1} a_{j, i-1} a_{j, i-1}^{*}-\sum_{j=1}^{i} a_{j i} a_{j i}^{*}\right):-v a_{i i}^{*} b_{i}-\chi_{i} a_{i i}^{*} . \\
& +\sum_{j=i+1}^{n} a_{i+1, j} a_{i j}^{*}-\sum_{j=1}^{i-1} a_{j, i-1} a_{j i}^{*}+\left(i+1-v^{2}\right) \dot{a}_{i i}^{*}, \\
H_{i}(z)= & 2: a_{i i} a_{i i}^{*}:+\sum_{j=1}^{i-1}\left(: a_{j i} a_{j i}^{*}:-: a_{j, i-1} a_{j, i-1}^{*}:\right) \\
& +\sum_{j=i+1}^{n}\left(: a_{i j} a_{i j}^{*}-: a_{i+1, j} a_{i+1, j}^{*}:\right)+v b_{i}+\chi_{i}, \\
F_{i}(z)= & a_{i i}+\sum_{j=i+1}^{n} a_{i j} a_{i+1, j}^{*}, \quad k=v^{2}-(n+1), \\
& \left(a_{i j}, a_{i j}^{*} \text { denote } a_{i j}(z), a_{i j}^{*}(z)\right)
\end{aligned}
$$

define the action of $L s l_{n+1}^{\Lambda}$ in $W_{\chi}$ with $\chi=\left(\chi_{1}, \ldots, \chi_{n}, v^{2}-(n+1)\right)$, the central charge is equal to $v^{2}-(n+1)$. We denote this module by $W_{\bar{\chi}, v}$, where $\bar{\chi}=\left(\chi_{1}, \ldots, \chi_{n}\right)$.

7.2. Co-twisted Verma Modules over Lsl $l_{2}^{A}$ with dominant weights are shown on the picture (Fig. 2). The points on the figures denote singular vectors in ${ }^{w} M_{\chi}$ or in its quotient, which correspond to singular vectors in the Verma module $M_{\chi}$. Interrelations between these vectors are expressed by the arrows. 


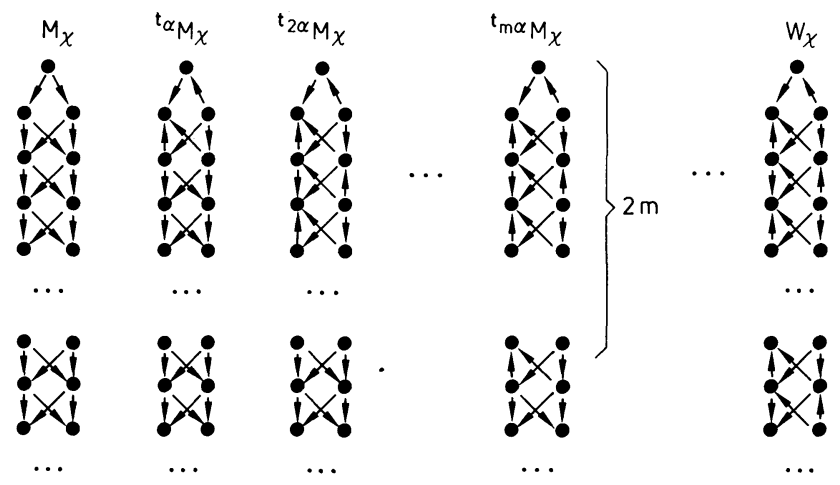

Fig. 2. Twisted Verma modules and Wakimoto module over $L s l_{2}^{\Lambda}$

The chain of arrows leads from one vector to another if and only if for any choice of vectors projecting under some factorizations into singular vectors, the latter vector is generated by the former.

In Fig. 2 the structure of the corresponding Wakimoto module is shown (the description of the structure of Wakimoto modules over $L s l_{2}^{A}$ was given in [19]). We see that this structure is "approximated" by the structures of $M_{\chi}^{w}$.

\subsection{Structure of Two-Sided BGG Resolutions over $\mathrm{Lsl}_{2}^{\Lambda}$ and $\mathrm{Lsl}_{3}^{\Lambda}$}

The affine Weyl group $S_{\text {aff }}\left(S L_{2}\right)$ is generated by two simple reflections $s_{0}$ and $s_{1}$. Let

$$
\begin{aligned}
& s^{(i)}=\underbrace{s_{1} s_{0} s_{1} \ldots s_{0 \text { or } 1}}_{-i \text { times }}, \quad i \leqq 0, \\
& s^{(i)}=\underbrace{s_{0} s_{1} s_{0} \ldots s_{0 \text { or } 1}}_{i \text { times }}, \quad i>0 .
\end{aligned}
$$

Then $R_{b}^{i}(\chi)=W_{s^{(i)} *}$. We want to give explicit formulae for the differential of $R_{b}^{*}(\chi)$.

In $[21,43,19]$ composition vertex operators

$$
B_{l_{1}, \ldots, l_{m}}\left(\beta_{1}, \ldots, \beta_{m} ; \gamma_{1}, \ldots, \gamma_{m}\right), \quad l_{i} \in \mathbb{Z}, \quad \beta_{i} \in \mathbb{C}, \quad \gamma_{i} \in \mathbb{C}, \quad \sum_{i=1}^{m} \gamma_{i}=0,
$$

were defined. $B_{l_{1}, \ldots, l_{m}}\left(\beta_{1}, \ldots, \beta_{m} ; \gamma_{1}, \ldots, \gamma_{m}\right)$ acts from $W_{\chi-\eta_{2} \beta_{1}, v, v}$ to $W_{\bar{\chi}, v}$.

Let $D_{l_{1}, \ldots, l_{m}}\left(\gamma_{1}, \ldots, \gamma_{m}\right)$ be the operator: $W_{\bar{\chi}-2 m, v} \rightarrow W_{\bar{\chi}, v}^{\prime}$ defined as follows:

$$
\begin{aligned}
D_{l_{1}, \ldots, l_{m}}\left(\gamma_{1}, \ldots, \gamma_{m}\right)= & \sum_{i_{1}, \ldots, i_{m} \in \mathbb{Z}} a\left(i_{1}\right) \ldots a\left(i_{m}\right) \\
& \times B_{l_{1}, \ldots, l_{m}}\left(\frac{1}{v}, \ldots, \frac{1}{v} ; \gamma_{1}, \ldots, \gamma_{m}\right), \quad \sum_{i=1}^{m} \gamma_{i}=0 .
\end{aligned}
$$

In [19] the following theorem is proved. 

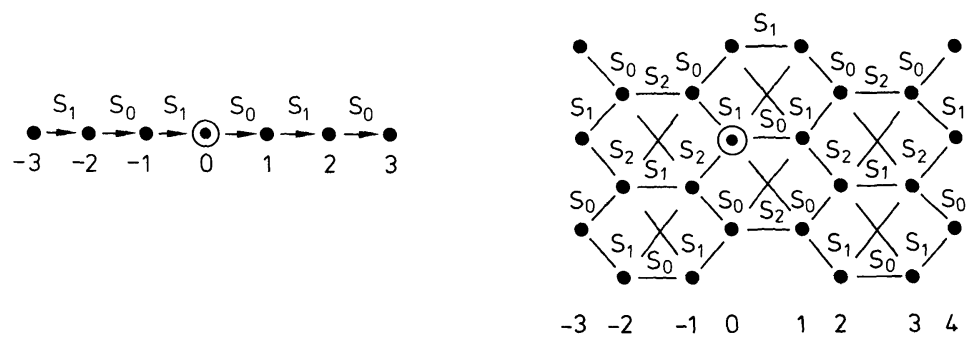

Fig. 3a, b. Two-sided BGG resolutions over $L s l_{2}^{\Lambda}$ and $L s l_{3}^{\Lambda}$. a $L s l_{2}^{\Lambda}$, b $L s l_{3}^{\Lambda}$

Theorem 6. Let $\gamma_{i}=\frac{1}{v^{2}}(\bar{\chi}-2(i-1))-l_{i}, i=1, \ldots, m$. If $\sum_{i=1}^{n} \gamma_{i}=0$ (Kac-Kazhdan equation [30]), then $D_{l_{1}, \ldots, l_{m}}\left(\gamma_{1}, \ldots, \gamma_{m}\right)$ is an intertwining operator between $W_{\bar{\chi}-2 m, v}$ and $W_{\bar{\chi}, v}$.

Now let $\chi=(m-1, l+m-2), l, m \in \mathbb{Z}, l, m>0$, by the integral dominant highest weight. Then the differential of $R_{b}^{*}(\chi) d_{i}: R_{b}^{i-1}(\chi) \rightarrow R_{b}^{i}(\chi)$ is given by [20],

$$
\begin{gathered}
d_{i}=D_{i, \ldots, i}\left(\gamma_{1}, \ldots, \gamma_{m}\right), \quad \gamma_{s}=\frac{m+1-2 s}{m+l}, \quad \text { if } i \text { is even }, \\
d_{i}=D_{i, \ldots, i}\left(\gamma_{1}, \ldots, \gamma_{l}\right), \quad \gamma_{s}=\frac{l+1-2 s}{m+l}, \quad \text { if } i \text { is odd } .
\end{gathered}
$$

The differential for the two-sided BGG resolution over other algebras may be also expressed via vertex operators. Two-sided BGG resolution over $L s l_{2}^{\Lambda}$ does exist for any highest weight $\chi=(\bar{\chi}, k)$ with $\bar{\chi}$ and $k$ rational and $k>-2[19,20]$.

The structure of the two-sided BGG resolution over $L s l_{2}^{\Lambda}$ and $L s l_{3}^{\Lambda}$ is shown in Fig. 3.

The points in the figure denote Wakimoto modules. The marked point denotes $W_{\chi}$. The arrows show the action of the differential. The weight of the module situated in the given point is equal to $s * \chi$, where $s$ is the product of simple reflections along the way from the marked point to the given point. Note that the pictures are composed of BGG resolutions over corresponding finite-dimension Lie algebras $s l_{2}$ and $s l_{3}$.

Two-sided BGG resolutions $R_{b}^{*}(\chi)$ over $L g^{\Lambda}$ may be used in WZW models for computations of the correlation functions on the torus and higher genus surfaces in integral representation, in the same way as in Felder's work [23].

Note that Felder's resolution over Virasoro algebra [23] is closely connected with our two-sided resolution over $L s l_{2}^{\Lambda}$ via the functorial correspondence between $L s l_{2}^{A}$-modules and modules over Virasoro algebra $[20,47]$.

\section{Appendix A. Semi-Infinite (Co)homology [16]}

Let $\mathscr{Z}$ be $\mathbb{Z}$-graded Lie algebra $\mathscr{Z}=\oplus_{i \in \mathbb{Z}} \mathscr{Z}_{i}, \operatorname{dim} \mathscr{Z}_{i}<\infty, \quad n=\mathscr{Z}_{1} \oplus \mathscr{Z}_{2} \oplus \ldots$, $b=\mathscr{Z}_{0} \oplus \mathscr{Z}_{-1} \oplus \ldots$ - its subalgebras. Let $M$ be such an $\mathscr{Z}$-module that the $n$-submodule generated by any vector of $M$ is finite-dimensional. 
Put $\mathscr{Z}^{*}=\bigoplus_{i \in \mathbb{Z}} \mathscr{Z}_{i}^{*}, \mathscr{Z}^{*}=n^{\perp} \oplus b^{\perp}$, where $\perp$ denotes the orthogonal complement. Choose a basis $\varphi_{i}, i \in \mathbb{Z}$ in $\mathscr{Z}$ and dual basis $\varphi_{i}^{*}, i \in \mathbb{Z}$ in $\mathscr{Z}^{*}$. Let $C l(\mathscr{Z})$ be a Clifford algebra with generators $\varphi_{i}, \varphi_{i}^{*}, i \in \mathbb{Z}$ and anticommutation relations: $\left[\varphi_{i}, \varphi_{j}\right]_{+}$ $=\left[\varphi_{i}^{*}, \varphi_{j}^{*}\right]_{+}=0,\left[\varphi_{i}, \varphi_{j}^{*}\right]_{+}=\delta_{i j}$. Introduce grading on $C l(\mathscr{Z})$, putting $\operatorname{deg} \varphi_{i}=-1$, $\operatorname{deg} \varphi_{i}^{*}=1$. Let $I$ be an irreducible representation of $C l(\mathscr{Z})$ with the vacuum vector annihilated by $\varphi_{i} \in n$ and $\varphi_{i}^{*} \in n^{\perp}$. The module $I$ inherits grading.

Put $\varphi=\sum: \varphi_{i} \varphi_{j}^{*} \varphi_{k}^{*}: c_{j k}^{i}$, where $c_{j k}^{i}$ are structural constants of $\mathscr{Z}$ and : : denotes normal ordering. Define the operator $T: M \otimes I \rightarrow M \otimes I$ as follows:

$$
T(m \otimes p)=m \otimes \varphi(p)+\sum \varphi_{i}(m) \otimes \varphi_{i}^{*}(p), \quad m \in M, p \in I .
$$

It is easy to see: $T^{2}=\mathrm{Id} \otimes \sum b_{i j} \varphi_{i}^{*} \varphi_{j}^{*}, b_{i j} \in \mathbb{C}$. The expression $\sum b_{i j} \varphi_{i}^{*} \varphi_{j}^{*}$ determines the 2 -form $\omega$ on $\mathscr{Z}$, and simple calculations show that $\omega$ is cocycle from $H^{2}(\mathscr{Z})$. If $H^{2}(\mathscr{Z})=0$, then $w=\delta v$, where $v=\sum r_{i} \varphi_{i}^{*}$ and $\delta$ is differential in the cohomological complex of $\mathscr{Z}$. Define the operator $d: M \otimes I \rightarrow M \otimes I$, putting $d=T-v$. Evidently, $d^{2}=0$. We obtain the complex $\{M \otimes I, d\}$, whose $i^{\text {th }}$ cohomologies are semi-infinite cohomologies of $\mathscr{Z}$ with coefficients in $M$ with respect to the decomposition $\mathscr{Z}=n \oplus b$. We denote them $H^{\infty / 2+i}(\mathscr{Z}, M)$.

Semi-infinite homologies are connected with semi-infinite homologies by the rule: $H_{\infty / 2+i}(\mathscr{Z}, M)=H^{\infty / 2-i}(\mathscr{Z}, M)$.

Our considerations may be applied to the finite dimensional Lie algebra $\mathscr{Z}$. The corresponding cohomology groups will be denoted $H_{s}^{i}(\mathscr{Z}, M)$ and called a shifted cohomology with respect to the decomposition $\mathscr{Z}=n \oplus b$.

\section{Appendix B. Modules on the Singular Hyperplane}

The singular hyperplane is the hyperplane $k=-c_{g}$ in $\widehat{h}^{*}$. The structure of $L g^{\Lambda}-$ modules, whose highest weights lie on the singular hyperplane, differ from the structure of other $\mathrm{Lg}^{\Lambda}$-modules. This is caused by the fact that Segal-Sugawara operators [28, 37] of $L g^{\Lambda}$ commute mutually and with $L g^{\Lambda}$ if $k=-c_{g}$. So they yield a great number of singular vectors in the Verma molecule. Let us consider a restricted Verma module $\bar{M}_{\left(\bar{\chi},-c_{g}\right)}=\bar{M}_{\bar{\chi}}$, which is the quotient of the Verma module $M_{\left(\bar{\chi},-c_{g}\right)}$ by a submodule, generated by all singular Segal-Sugawara vectors. In $[28,37,45]$ it is proved that in general a point of the singular hyperplane (that is if $\chi$ does not belong to other Kazhdan-Lusztig hyperplanes [30]) $\bar{M}_{\bar{\chi}}$ is irreducible and hence isomorphic to the restricted Wakimoto module $\bar{W}_{\bar{\chi}}$ defined in 5.1 .

In [19] we used explicit formulae for $\bar{W}_{\bar{\chi}}$ for the study of the structure of a restricted Verma module. Here we sketch our main results.

First of all we consider $\bar{W}_{\bar{\chi}}$ and $\bar{M}_{\bar{\chi}}$ over $\operatorname{Lsl}_{2}^{\Lambda}\left(c_{g}=2\right)$.

Theorem B.1 [19]. If $\bar{\chi}=0,1,2, \ldots$ then $\bar{M}_{\bar{\chi}}$ is isomorphic to $\bar{W}_{\bar{\chi}}$ and contains the unique singular vector of degree- $(\bar{\chi}+1) \alpha_{1}$, the quotient by the submodule generated by this singular vector being irreducible.

If $\bar{\chi}=-2,-3, \ldots$, then $\bar{M}_{\bar{\chi}}$ is isomorphic to $\bar{W}_{\bar{\chi}}^{*}$ and contains the unique singular vector of degree $(+\bar{\chi}+1) \alpha_{0}$, the quotient by the submodule generated by this singular vector being irreducible.

If $\bar{\chi} \neq 0,1,2$ and $\bar{\chi} \neq-2,-3, \ldots$ then $\bar{M}_{\bar{\chi}}$ is irreducible and isomorphic to $\bar{W}_{\bar{\chi}}$. 
Note that Malikov has proved this result by other means [38].

For a general affine Kac-Moody algebra $L^{A}{ }^{A}$ we have proved the theorem about the structure of $\bar{M}_{\bar{\chi}}$, if $\bar{\chi}$ is projective. We call $\bar{\chi}$ projective if the Verma module $M_{\bar{\chi}}$ over $g$ is projective in the category $\mathcal{O}$ [5]. It means that $M_{\bar{\chi}}$ is not contained in another Verma module over $g$ as a proper submodule. In particular, if $M_{\bar{\chi}}$ is irreducible and is not contained in another Verma module, then $\chi$ is projective, and the dominant integral weight is projective.

Theorem B.2 [19]. If weight $\bar{\chi}$ is projective, then the singular vectors of $\bar{M}_{\bar{\chi}}$ over $\mathrm{Lg}^{\mathrm{A}}$ coincide with singular vectors of $M_{\bar{\chi}}$ over $\mathrm{g}$, and also $\bar{M}_{\bar{\chi}}$ is isomorphic to $\bar{W}_{\bar{\chi}}$. In particular, if $M_{\bar{\chi}}$ is irreducible and is not contained in another Verma module then $\bar{M}_{\bar{\chi}}$ and $\bar{W}_{\bar{\chi}}$ are irreducible (and mutually isomorphic).

We also have the conjecture about non-projective highest weight modules.

Let $\bar{\chi}_{1}, \ldots, \bar{\chi}_{l}$ be highest weights of Verma modules over $g$ such that $M_{\bar{\chi}_{i}} \subset M_{\bar{\chi}}$ or $M_{\bar{\chi}} \subset M_{\bar{\chi}_{i}}$ or there is $\bar{\chi}_{j}$, such that $M_{\bar{\chi}_{i}} \subset M_{\bar{\chi}_{j}}, M_{\bar{\chi}} \subset M_{\bar{\chi}_{j}}$. Let $\bar{\chi}_{\max }$ be the maximal weight among $\bar{\chi}_{1}, \ldots, \bar{\chi}_{1}, \bar{\chi}$. Then $\bar{\chi}_{\max }$ is the projective weight.

Conjecture B.3 [19]. The number of singular vectors of $\bar{M}_{\bar{\chi}}$ coincide with the number of singular vectors of $\bar{M}_{\bar{\chi}_{\max }}$. In particular, if $\bar{\chi}=s * \bar{\chi}_{0}$, where $\bar{\chi}_{0}$ is the dominant integral weight, then the number of singular vectors in $\bar{M}_{\bar{\chi}}$ is equal to the order of the Weyl group $S(G)$.

We also conjecture the acyclic resolution consisting of restricted Verma modules. This resolution seems to be the Cousin-Grothendieck resolution of the invertible sheaf on the manifold $\widetilde{L(B)}$ with respect to Schubert filtration.

Conjecture B.4 [19]. There is an acyclic resolution $A^{i}(\bar{\chi})(\bar{\chi}$ is the dominant integral weight) of $\mathrm{Lg}^{A}$-modules with central charge $-c_{g}$, so that $A^{i}=\underset{\substack{l t(s)=-i \\ s \in S_{\text {aff }}}}{\oplus} \bar{M}_{s(\bar{\chi}+\varrho)-\varrho}$.

Acknowledgements. We would like to thank A. A. Beilinson and D. B. Fuchs for helpful discussions.

\section{References}

1. Beilinson, A.: Localization of representations of reductive Lie algebras. In: Proceedings of ICM, Warsawa 1984, Vol. 2, pp. 699-710

2. Beilinson, A., Bernstein, J.: Localization de g-modules. C.R. Acad. Sci. Paris 192, 15-18 (1981)

3. Beilinson, A., Bernstein, J.: Generalization of a theorem of Casselman. Report on Utah Conference on representation theory, April 1982. In: Representation theory of reductive groups. Trombi, P. (ed.) DM 40. Boston: Birkhäuser 1983

4. Bernstein, J.: Lectures on $D$-modules (unpublished)

5. Bernstein, J., Gelfand, I., Gelfand, S.: On the category of $g$-modules. Funkt. Anal. Prilozhen. 10, 1-8 (1976) (in Russian)

6. Bernstein, J., Gelfand, I., Gelfand, S.: Schubert cells and cohomology of spaces $G / P$. Usp. Mat. Nauk (= Russ. Math. Surv.) 28, 3-26 (1973) (in Russian)

7. Bernstein, J., Gelfand, I., Gelfand, S.: Differential operators on the base affine space and a study of $g$-modules. In: Lie groups and their representations, pp. 21-64. New York: Wiley 1975

8. Borel, A.: Kählerian coset spaces of semi-simple Lie groups. Proc. Natl. Acad. USA 40, 1147-1151 (1954) 
9. Bott, R.: Homogeneous vector bundles. Ann. Math. 66, 203-248 (1957)

10. Brylinsky, J.L., Kashiwara, M.: Kazhdan-Lusztig conjecture and holonomic systems. Invent. Math. 64, 487-410 (1981)

11. Conley, C.C., Zehnder, E.: Morse type index theorem for flows and periodic solutions for hamiltonian equation. Commun. Pure and Appl. Math. 34, 207-253 (1984)

12. Date, E., Jimbo, M., Kashiwara, M., Miwa, T.: Transformation groups for soliton equations. In: Proceedings of RIMS symposium. Jimbo, M., Miwa, T. (eds.), pp. 39-120. Singapore: World Scientific 1983

13. Deodhar, V., Gabber, O., Kac, V.: Structure of some categories of representations of infinitedimensional Lie algebras. Adv. Math. 45, 92-116 (1982)

14. Dotsenko, Vl.S., Fateev, V.A.: Conformal algebra and multipoint correlation functions in $2 D$ statistical models. Nucl. Phys. B 240, F 312, 312-348 (1984)

15. Dotsenko, Vl.S., Fateev, V.A.: Private communications

16. Feigin, B.L.: Semi-infinite homology of Kac-Moody and Virasoro Lie algebras. Usp. Mat. Nauk (= Russ. Math. Surv.) 39, 195-196 (1984) (in Russian)

17. Feigin, B.L.: On the cohomology of the Lie algebra of vector fields and of the current algebra. Sel. Math. Sov. 7, 49-62 (1988)

18. Feigin, B.L., Frenkel, E.V.: The family of representations of affine Lie algebras. Usp. Math. Nauk (= Russ. Math. Surv.) 43, 227-228 (1988) (in Russian)

19. Feigin, B.L., Frenkel, E.V.: Representations of affine Kac-Moody algebras and bosonization. To be published in: V. G. Knizhnik Memorial volume. Singapore: World Scientific 1989

20. Feigin, B.L., Frenkel, E.V.: Representations of affine Kac-Moody algebras, bosonization and resolutions. To be published in Lett. Math. Phys. 19, N3 (1990)

21. Feigin, B.L., Fuchs, D.B.: Representations of the Virasoro algebra. To be published in: Representations of infinite-dimensional Lie groups and Lie algebras. New York: Gordon and Breach 1989

22. Feigin, B.L., Tsygan, B.L.: Cohomology of generalized Jakobean matrices. Funct. Anal. Prilozhen. 17, 86-87 (1983)

23. Felder, G.: BRST approach to minimal models. Nucl. Phys. B 317, 215-236 (1989)

24. Floer, A.: Proof of Arnold conjecture for surfaces and generalization to certain Kähler manifolds. Duke Math. J. 53, 1-32 (1986)

25. Fuchs, D.B.: Cohomology of infinite-dimensional Lie algebras. New York: Plenum Press 1986

26. Garland, H., Lepowsky, J.: Lie algebra homology and the Macdonald-Kac formulas. Invent. Math. 34, 37-76 (1976)

27. Gerasimov, A., Marshakov, A., Morosov, A., Olshanetsky, M., Shatashvili, S.: Moscow preprint, 1989

28. Hayashi, T.: Sugawara construction and Kac-Kazhdan conjecture. Invent. Math. 94, 13-52 (1988)

29. Kac, V.G.: Infinite-dimensional Lie algebras. Boston, MA: Birkhäuser 1983

30. Kac, V.G., Kazhdan, D.: Structure of representations with highest weight of infinitedimensional Lie algebras. Adv. Math. 34, 97-108 (1979)

31. Kac, V., Peterson, D.: Unpublished, Kac, V.G.: Constructing groups associated to infinitedimensional Lie algebras. In MSRI Publ., 4. Berlin, Heidelberg, New York: Springer 1985

31a. Kac, V., Peterson, D.: Spin and wedge representations of infinite-dimensional Lie algebras and groups. Proc. Natl. Acad. Sci. USA 78, 3308-3312 (1981)

32. Kac, V., Todorov, I.: Superconformal current algebras and their unitary representations. Commun. Math. Phys. 102, 337-347 (1985)

33. Kazhdan, D., Lusztig, G.: Representation of Coxeter groups and Hecke algebras. Invent. Math. 53, 165-184 (1979)

34. Kazhdan, D., Lusztig, G.: Schubert varieties and Poincaré duality. Proc. Symp. Pure Math. 36, 185-203 (1980)

35. Kempf, G.: Cousin-Grothendieck complex of induced representations. Adv. Math. 29, 310-396 (1978) 
36. Kostant, B., Kumar, S.: The nil Hecke ring and cohomology of G/P for a Kac-Moody group. Adv. Math. 62, 187-237 (1986)

37. Malikov, F.: Singular vectors in Verma modules over affine algebras. Funct. Anal. Prilozhen. 23, 76-77 (1989)

38. Malikov, F.: Verma modules over affine rank 2 algebras. To appear in Algebra and Analysis (1989) (in Russian)

39. Peterson, D., Kac, V.: Infinite flag varieties and conjugacy theorem. Proc. Natl. Acad. USA 80, 1778-1782 (1983)

40. Pressley, A., Segal, G.: Loop groups. Oxford: Clarendon Press 1987

41. Rocha-Caridi, A., Wallach, N.: Projective modules over graded Lie algebras. Math. Z. 180, 151-177 (1982)

42. Tate, J.: Residues of differentials on curves. Ann. Sci. E.N.S. 4 Ser., t. 1, 149-159 (1968)

43. Tsuchiya, A., Kanie, Y.: Fock space representations of the Virasoro algebra - Intertwining operators. Publ. RIMS, Kyoto Univ. 22, 259-327 (1986)

44. Wakimoto, M.: Fock representations of affine Lie algebra $A_{1}^{(1)}$. Commun. Math. Phys. 104, 604-609 (1986)

45. Wallach, N.: A Class of non-standard modules for affine Lie algebras. Math. Z. 196, 303-313 (1987)

46. Zamolodchikov, A.: Unpublished

47. Bershadsky, M., Ooguri, H.: Hidden $s l(n)$ symmetry in conformal field theory, IASSNS HEP-89/09

48. Bernard, D., Felder, G.: Fock representations and BRST cohomology of sl(2) current algebra, ETH-TH/89-26

49. Bouwknegt, P., McCarthy, J., Pilch, K.: MIT preprint CTP \#1797

Communicated by L. Alvarez-Gaumé

Received June 6, 1989

Note added in proof. After this paper had been accepted for publication, we received preprint [48], where a two-sided BGG resolution (BRST complex) was constructed for $L s l_{2}^{A}$. The formulas obtained in [48] coincide with ours from 7.3. In [49] certain explicit formulas for the differential of two-sided BGG resolution (BRST operator) were given using vertex operators introduced in [19]. We would like to thank P. Bouwknegt and J. McCarthy for interesting discussions. 
